\title{
Nonlinear dynamics of viscous droplets
}

\author{
By E. BECKER†, W. J. HILLER AND T. A. KOWALEWSKI \\ Max-Planck-Institut für Strömungsforschung, Bunsenstrasse 10, D-37073 Göttingen, Germany
}

(Received 9 July 1992 and in revised form 21 May 1993)

Nonlinear viscous droplet oscillations are analysed by solving the Navier-Stokes equation for an incompressible fluid. The method is based on mode expansions with modified solutions of the corresponding linear problem. A system of ordinary differential equations, including all nonlinear and viscous terms, is obtained by an extended application of the variational principle of Gauss to the underlying hydrodynamic equations. Results presented are in a very good agreement with experimental data up to oscillation amplitudes of $80 \%$ of the unperturbed droplet radius. Large-amplitude oscillations are also in a good agreement with the predictions of Lundgren \& Mansour (boundary integral method) and Basaran (Galerkin-finite element method). The results show that viscosity has a large effect on mode coupling phenomena and that, in contradiction to the linear approach, the resonant mode interactions remain for asymptotically diminishing amplitudes of the fundamental mode.

\section{Introduction}

The study of droplet dynamics can be traced back to the early work of Lord Rayleigh (1879). Lamb (1932, pp. 473-475, 639-641) extended the inviscid linear analysis, including weak dissipative effects, and later Chandrasekhar (1961, pp. 466-477) solved the full viscous problem by mode analysis. Subsequently, Prosperetti $(1977,1980 a, b)$ noted that the linear theory left open some questions about the initial value problem. For example, the amplitudes of Chandrasekhar's eigenfunctions are unknown for the oscillations of a deformed droplet starting from rest. The analysis of initial vorticity generation, first performed by Prosperetti, is repeated here (in Appendix A), using a new formulation for the velocity field, leading to an analytic solution for the problem.

Although during the last decades nonlinear droplet dynamics has become the object of several theoretical and experimental investigations, it remains one of the classical problems in hydrodynamics for which a complete theoretical solution is lacking. However, theoretical models describing particular aspects of nonlinear droplet dynamics have become very important in several applications, for example in measuring fluid properties like surface tension and viscosity (Hiller \& Kowalewski 1989; Becker, Hiller \& Kowalewski 1991) or in nuclear physics (Brosa \& Becker 1988; Brosa et al. 1989). In particular, the possibility of non-intrusive measurements of dynamic surface tension by the oscillating droplet method is of great interest for the determination of physico-chemical properties of liquid mixtures (Defay \& Pétré 1971;

$†$ Present address: Institut für Atmosphärenphysik an der Universität Rostock e.V., Schloßstr. 4-6, D-18221 Kühlungsborn, Germany.

$\ddagger$ On leave of absence from the Institute of Fundamental Technological Research, Polish Academy of Sciences, PL-00-049 Warszawa, Poland. 
Stückrad, Hiller \& Kowalewski 1993). Our experimental investigations of droplet oscillations have shown that linear theory as well as nonlinear inviscid theory have a very limited range of applicability in the interpretation of experimental results. Hence, our present interest is concentrated on the development of a nonlinear model including viscous effect which allows an easy analysis of experimental data enabling a calculation of the dynamic surface tension of the investigated liquids.

The existing theoretical models describing nonlinear droplet dynamics either neglect viscosity (Tsamopoulos \& Brown 1983; Natarajan \& Brown 1987) or, whilst taking viscosity into account, use a strictly numerical approach (Lundgren \& Mansour 1988; Basaran 1992). The limitations of existing techniques have been widely discussed by Patzek et al. (1991). It seems that two methods, namely the boundary integral method applied by Lundgren \& Mansour (1988) and Galerkin-finite element method by Basaran (1992) offer a reasonable approach to nonlinear and viscous droplet dynamics. However these methods, aside from their numerical complexity, have limited practical applicability. The boundary integral methods, as was shown by Patzek et al. (1991), cannot model droplet oscillations when the effects of viscosity are in the range that is physically of interest. The finite element methods are limited at low viscosities (higher Reynolds numbers require fine discretizations and long computational time).

The new approach presented here offers the possibility of analysing nonlinear droplet dynamics for a wide range of nondimensional viscosity. Furthermore, it allows monitoring of the systematic errors of the algorithm by means of physically justified integrals.

The present model of droplet oscillations ( $(4)$ uses the mode expansion method with appropriate modes of the linear problem and takes into account all nonlinearities as well as viscosity. This method is akin to the work of Boberg \& Brosa (1988) who analysed the transition to turbulence in a tube flow with the help of a corresponding mode expansion. The existence of stationary boundary conditions in Boberg \& Brosa's problem allowed them to use Galerkin's method to deduce their system of ordinary differential equations. In the case of a free boundary problem the modes do not satisfy the boundary conditions a priori. Therefore, a direct application of semi-analytical methods becomes difficult. Hence, the problem of deriving an appropriate system of ordinary differential equations is solved by the use of the standard variational principle of Gauss. This, one of the most general principles of classical mechanics, seems to be well suited to the analysis of nonlinear droplet oscillations, since it offers the straightforward possibility of treating the boundary conditions as additional constraints on the Navier-Stokes (or the vorticity) equations. For special cases, if highwavenumber modes of the droplet oscillation are strongly excited, the method proposed in the present paper may become less appropriate compared with the aforementioned pure numerical solvers of Lundgren \& Mansour or Basaran. However, this limitation of our approach has no effect on its application to physical experiments with a free oscillating droplet, where amplitudes of the oscillation modes are strongly related to their linear damping constants. On the other hand our method is close to the physics of nonlinear droplet oscillations, describing its dynamics in terms of the natural degrees of freedom. This has been established both by the comparison of the computed droplet oscillations with experimental data given in Becker et al. (1991) and repeating some droplet trajectories generated by Lundgren \& Mansour (1988) and Basaran (1992). 


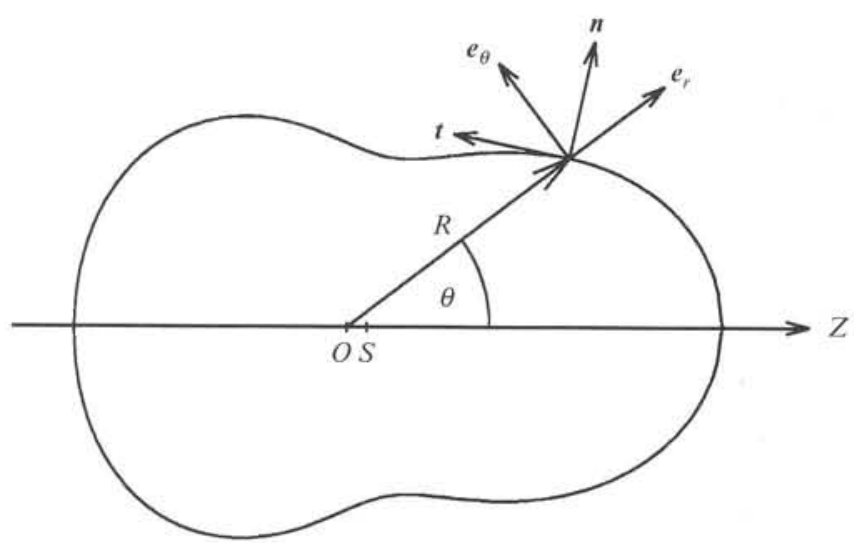

FIGURE 1. A droplet cross-section described by the surface parametrization (2.1). The unit vectors of the coordinate system as well as normal and tangential unit vectors of the surface are displayed. The origin of the coordinate system is denoted by $O$, and the centre of mass of the droplet by $s$.

\section{Formulation of the problem}

We consider an incompressible droplet of equilibrium radius $r_{0}$, density $\rho$, uniform surface tension $\sigma$ and kinematic viscosity $\nu$, which is freely oscillating in a medium of negligible density and viscosity.

In the mathematical description, limited to axisymmetric droplet dynamics, spherical coordinates $(r, \theta)$ are used where $r$ is the distance from the system origin and $\theta$ is the meridian angle measured from the axis of symmetry, $z$. As in our previous analysis (Becker et al. 1991), we assume that the radial distance $R(\theta, t)$ from the origin of the coordinate system to the droplet surface can be expanded in a series of Legendre polynomials $P_{l}(\cos \theta)$ :

$$
R(\theta, t)=r_{0}\left\{a_{0}\left(a_{2}, \ldots, a_{l_{0}}\right)+\sum_{l=2}^{l_{0}} a_{l}(t) P_{l}(\cos \theta)\right\} .
$$

Figure 1 illustrates the geometry. Assuming constant liquid density, $R(\theta, t)$ always encloses the same volume:

$$
\frac{4}{3} \pi r_{0}^{3}=2 \pi \int_{-1}^{1} \frac{1}{3} R^{3}(\theta, t) \mathrm{d} \cos \theta .
$$

This condition leads to a cubic equation defining the dimensionless mean droplet radius $a_{0}$ as a function of the surface parameters $a_{2} \ldots a_{t_{0}}$. It turns out that $a_{0}$ is always less than or equal to one. The droplet shape is uniquely described by the parameters $a_{2} \ldots a_{l_{0}}$. They can be interpreted as dimensionless amplitudes of standing waves, with I periods, on strings encircling the cross-section of the droplet. These waves oscillate independently of each other only in the linear case.

The unit vectors normal $(n)$ and tangential $(t)$ to the droplet surface are given by the following formulae:

$$
\boldsymbol{n}=\frac{R \boldsymbol{e}_{r}-\partial_{\theta} R \boldsymbol{e}_{\theta}}{\left[R^{2}+\left(\partial_{\theta} R\right)^{2}\right]^{\frac{1}{2}}}, \quad t=\frac{\partial_{\theta} R \boldsymbol{e}_{r}+R \boldsymbol{e}_{\theta}}{\left[R^{2}+\left(\partial_{\theta} R\right)^{2}\right]^{\frac{1}{2}}},
$$

where $e_{r}$ and $e_{\theta}$ are unit vectors in the radial and the polar directions, respectively.

In our experimental analysis (Becker et al. 1991) the surface parametrization (2.1) yields the amplitudes $a_{2} \ldots a_{l_{0}}$, the equivolumetric radius $r_{0}$ and the position of the 
coordinate system. These parameters are fitted to the observed droplet shape. However, the origin $O$ of the coordinate system does not need to coincide with the centre of mass $s$ which may move along the symmetry axis $z$. The displacement $s$ of the centre of mass depends on the surface deformation and in the chosen coordinate system it is given by

$$
s\left(a_{2} \ldots a_{l_{0}}\right)=\frac{3}{8} r_{0} \int_{-1}^{1} \cos \theta\left(a_{0}\left(a_{2} \ldots a_{l_{0}}\right)+\sum_{l=2}^{l_{0}} a_{l} P_{l}(\cos \theta)\right)^{4} \mathrm{~d} \cos \theta .
$$

The parameterization (2.1) does not include the term with index $l=1$ because in our experimental analysis this term does not represent an additional degree of freedom for the description of the surface. The amplitude $a_{1}$ which in the linear theory describes a pure translational motion cannot be separated out in the experimental analysis. Obviously it is possible to let $a_{1}$ be different from zero and to require the centre of mass to coincide with the origin of coordinates. This gives a second relation in addition to (2.2), which allows the elimination of $a_{1}$ as a function of $a_{2} \ldots a_{l_{0}}$. Both descriptions become equivalent if $s / r_{0}$ (or $\left.a_{1}\right)$ is small. In practice the value of $\left|s / r_{0}\right|$ remains below 0.01 in our experimental and computational analysis.

In the non-inertial coordinate system the Navier-Stokes equation for incompressible fluids has the following form:

$$
\partial_{t} v+(v \cdot \nabla) v-\ddot{s} e_{z}=-\nabla p / \rho-v \nabla \times \nabla \times v,
$$

where $v$ denotes the velocity field, $e_{z}$ the unit vector in the $z$-direction and $p$ the pressure. The acceleration of the coordinate system with respect to the centre of mass is equal to $-\ddot{s}$. In the case of constant density $\rho$ the governing equation (2.5) is equivalent to the vorticity equation $(V E)$ :

$$
\partial_{t} \boldsymbol{w}=\boldsymbol{\nabla} \times(\boldsymbol{v} \times \boldsymbol{w})-\nu \boldsymbol{\nabla} \times \boldsymbol{\nabla} \times \boldsymbol{w}, \quad \boldsymbol{w}=\boldsymbol{\nabla} \times \boldsymbol{v} .
$$

Surface motion and flow velocity are coupled by the kinematic boundary condition $(K B C)$ :

$$
v \cdot\left(R e_{r}-\partial_{\theta} R e_{\theta}\right)=R \partial_{t} R, \quad r=R .
$$

The driving force of droplet oscillations, namely the surface tension, acts perpendicularly to the free surface. Therefore, on the surface of the tangential stress of the flow vanishes, and the normal stress balances the driving force. The tangential stress condition (TSC) and dynamic boundary condition $(D B C)$ become

$$
\begin{aligned}
& (\boldsymbol{T} \boldsymbol{n}) \cdot t=0, \quad r=R, \\
& (\boldsymbol{T} \boldsymbol{n}) \cdot \boldsymbol{n}=2 \sigma H, \quad r=R .
\end{aligned}
$$

$\boldsymbol{T}$ is the Newtonian stress tensor and $H$ the mean curvature of the droplet surface. The left-hand side of (2.8) contains only friction terms which are known from $v$. The pressure $p$ contained in the left-hand side of the dynamic boundary condition (2.9) is given by line integration of the Navier-Stokes equation (2.5).

Computational results are non-dimensionalized using $r_{0}$ and $T_{0}=\left(\rho r_{0}^{3} / 3 \sigma\right)^{\frac{1}{2}}$ as scale factors for length and time.

\section{Linear oscillations of a viscous droplet}

A simple analytical method of finding partial solutions of linearized Navier-Stokes problems was given by Brosa $(1986,1988)$. These partial solutions applied below to describe linear oscillations of a viscous droplet were found to be also useful for 
investigations of the corresponding nonlinear problem (\$4). In Appendix A results obtained previously by Prosperetti $(1977,1980 a)$ are recapitulated to illustrate the advantages of Brosa's modes ansatz.

The partial solutions of the linearized Navier-Stokes equation in the case of rotational symmetry and free boundary conditions are given in the following form (Appendix B):

and

$$
\begin{gathered}
\boldsymbol{v}_{l}=\mathrm{e}^{-\lambda t}\left[b_{l}^{0} \nabla \times \nabla \times\left\{\boldsymbol{r} j_{j}\left[(\lambda / \nu)^{\frac{1}{2}} r\right] P_{L}(\cos \theta)\right\}+c_{l}^{0} \nabla\left\{\left(r / r_{0}\right)^{l} P_{l}(\cos \theta)\right\}\right], \\
p_{l}=\rho \lambda \mathrm{e}^{-\lambda t} c_{l}^{0}\left(r / r_{0}\right)^{l} P_{l}(\cos \theta) .
\end{gathered}
$$

Owing to linearity, $a_{l}$ will have the same time dependence as $v_{l}$ and $p_{l}$ :

$$
a_{l}(t)=a_{l}^{0} \mathrm{e}^{-\lambda t} .
$$

Inserting (3.1)-(3.3) into the linearized forms of the boundary conditions (2.7)-(2.9) and making use of the orthogonality of the Legendre polynomials one obtains a homogeneous system of equations for the amplitudes $b_{l}^{0}, c_{l}^{0}$ and $a_{l}^{0}$. The condition of non-trivial solutions for this system yields after several algebraic transformations

$$
\begin{gathered}
\operatorname{det}(x)=\left[4 l(l-1)(l+2)-2 x^{2}+2 \frac{\alpha^{4}}{x^{2}}\right] \frac{\mathrm{d}}{\mathrm{d} x}\left(x j_{l}(x)\right) \\
+\left[-4 l(l+2)\left(l^{2}-1\right)+4 l^{2} x^{2}-x^{4}+\alpha^{4}-2(l+1) \alpha^{4} / x^{2}\right] j_{l}(x)=0, \\
x:=(\lambda / \nu)^{\frac{1}{2}} r_{0}, \\
\alpha^{4}:=-\Omega^{2}\left(r_{0}^{2} / \nu\right)^{2} \\
\Omega^{2}:=\left(\sigma / \rho r_{0}^{3}\right) l(l-1)(l+2) .
\end{gathered}
$$

The characteristic equation (3.4) is equivalent to that given by Chandrasekhar (1961, chapter X, equation (280)). $\Omega$ is the eigenfrequency in the case of an ideal fluid. The existence of periodic solutions depends on the value of $\left|\alpha^{2}\right|$ which plays the role of an l-dependent Reynolds number. In the asymptotic case of small viscosity $\left(\left|\alpha^{2}\right| \rightarrow \infty\right)$ and oscillatory motion ( $x$ is complex) an analytical solution of (3.4), namely Lamb's irrotational approximation

$$
\lambda=\delta \pm \mathrm{i}\left(\Omega^{2}-\delta^{2}\right)^{\frac{1}{2}}, \quad \delta=\frac{(2 l+1)(l-1) v}{r_{0}^{2}},
$$

follows. In general the complex roots of $\operatorname{det}(x)$ must be found numerically. Figure 2 shows the results for $l=2$. For large values of $\left|\alpha^{2}\right|$ two conjugate roots are obtained. When $\left|\alpha^{2}\right|$ increases from $\left|\alpha_{\text {erit }}^{2}\right|$ to infinity, they form two branches in the complex plane of $x$. These branches represent weakly damped oscillations. In the limit $\left|\alpha^{2}\right| \rightarrow \infty$ the damping vanishes, i.e. the relation $|\operatorname{Re}(x) / \operatorname{Im}(x)|$ becomes unity. With decreasing $\left|\alpha^{2}\right|$ damping increases until both branches combine at $x=x_{\text {crit }}\left(\left|\alpha^{2}\right|=\left|\alpha_{\text {crit }}^{2}\right|\right)$, and an oscillatory motion is no longer possible. Further decrease of $\left|\alpha^{2}\right|$ leads to two real roots, describing aperiodic decay of droplet deformations. In the first case $x$ tends to zero with $\left|\alpha^{2}\right|$ whereas in the second $x$ tends to $x_{0}$. In addition to this pair of solutions, which depends strongly upon $\left|\alpha^{2}\right|$, there exists an infinite spectrum of nearly constant real roots. These represent internal vortices of the droplet flow and give rise to strongly dissipative modes. In figure 2 black squares mark the first three solutions. It is surprising that these roots and therefore the corresponding velocity fields vary only weakly as $\left|\alpha^{2}\right|$ changes from zero to infinity. The zero-maps for the higher wavenumbers $(l>2)$ look similar to that in figure 2.

In contrast to the inviscid analysis the characteristic equation (3.4) has non-trivial solutions for $l=1$. This infinite set of real roots - not mentioned by other authors - 


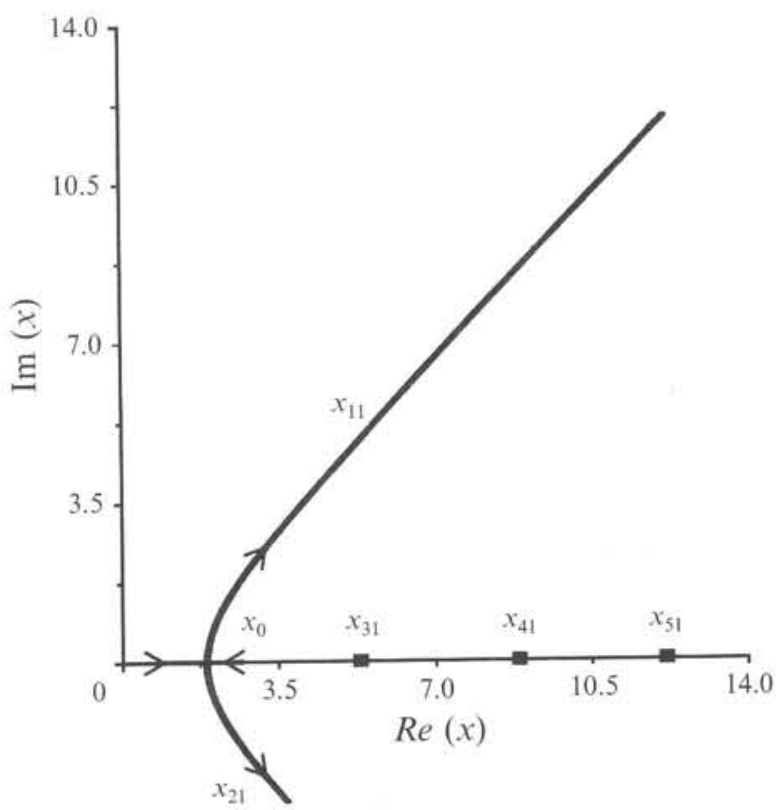

FIGURE 2. Map of roots of the characteristic equation (3.4) for polar wavenumber $l=2$. The arrows indicate the direction of increasing $\left|\alpha^{2}\right|$.

\begin{tabular}{ccccccccc}
\hline$l$ & $\left|\alpha_{\text {crit }}^{2}\right|$ & $x_{\text {crit }}$ & $x_{0}$ & $x_{11}$ & $x_{2 t}$ & $x_{3 l}$ & $x_{4 l}$ & $x_{51}$ \\
1 & - & - & - & 3.87 & 7.44 & 10.7 & 13.9 & 17.1 \\
- & - & - & - & $x_{3 t}$ & $x_{4 l}$ & $x_{5 t}$ & $x_{6 t}$ & $x_{7 l}$ \\
2 & 3.69 & 1.89 & 2.67 & 5.32 & 8.86 & 12.2 & 15.4 & 18.6 \\
3 & 8.82 & 2.85 & 4.0 & 6.63 & 10.2 & 13.6 & 16.8 & 20.0 \\
4 & 15.4 & 3.71 & 5.16 & 7.89 & 11.5 & 14.9 & 18.2 & 21.4 \\
5 & 23.6 & 4.51 & 6.25 & 9.11 & 12.8 & 16.2 & 19.6 & 22.8 \\
6 & 33.2 & 5.29 & 7.29 & 10.3 & 14.1 & 17.5 & 20.9 & 24.2 \\
7 & 44.4 & 6.06 & 8.32 & 11.5 & 15.3 & 18.8 & 22.2 & 25.5 \\
8 & 57.1 & 6.81 & 9.33 & 12.6 & 16.5 & 20.1 & 23.5 & 26.9
\end{tabular}

TABLE 1. $\left|\alpha_{\text {erit }}^{2}\right|, x_{\text {crit }}, x_{0}$ and the roots of the first five strongly dissipative modes for given polar wavenumbers $l=1 \ldots 8$. Owing to the weak dependence on $\left|\alpha^{2}\right|$ (for $l \geqslant 2$ ) the roots of the strongly dissipative modes are only approximate values.

is independent of $\left|\alpha^{2}\right|$ and gives rise to strongly dissipative modes, describing internal velocity fields which leave the droplet surface at rest. In fact there are no $a_{1}$ deformations.

Summarizing, we obtain the discrete spectrum of eigenvalues

$$
\left\{\lambda_{i l} \mid i=1,2, \ldots ; \quad l=1,2, \ldots\right\},
$$

characterized by radial and polar wavenumbers $i$ and $l$. Each pair of complex solutions is defined as $\lambda_{1 l}$ and $\lambda_{2 l}$ where the imaginary part of $\lambda_{1 l}$ is supposed to be positive. The additional damping constants of the strongly dissipative modes are enumerated with radial wavenumbers $i \geqslant 3$. In the case of $\left|\alpha^{2}\right|<\left|\alpha_{\text {crit }}^{2}\right|$ or $l=1$ only real eigenvalues occur; they are numbered with monotonically increasing numbers $i \geqslant 1$. The roots $\left\{x_{i l}\right\}$ of (3.4) are labelled in the same way (see figure 2). Table 1 displays the most important values for polar numbers $l=1 \ldots 8$. 
The amplitudes $b_{i l}^{0}, c_{i l}^{0}$ and $a_{i l}^{0}$ of the mode system can be found from the homogeneous equation system formed by the linerized boundary conditions. As one of the amplitudes is arbitrary we can choose $b_{i l}^{0}$ in a way that normalizes the partial solutions of the vorticity:

$$
\begin{gathered}
w_{i l}(r, \theta) \boldsymbol{e}_{\phi}=b_{i l}^{0} \boldsymbol{\nabla} \times \boldsymbol{\nabla} \times \boldsymbol{\nabla} \times\left\{r j_{l}\left(x_{i l} \frac{r}{r_{0}}\right) P_{l}(\cos \theta)\right\} \\
=e_{\phi} b_{i l}^{0}\left(\frac{x_{i l}}{r_{0}}\right)^{2} j_{l}\left(x_{i l} \frac{r}{r_{0}}\right) P_{l}^{\prime}(\cos \theta) \sin \theta, \\
\int_{-1}^{+1} \mathrm{~d} \cos \theta \int_{0}^{r_{0}} r^{2} \mathrm{~d} r w_{i l}^{*} w_{i l}:=\frac{r_{0}^{3}}{T_{0}^{2}} .
\end{gathered}
$$

In (3.10) $\boldsymbol{e}_{\phi}=\boldsymbol{e}_{r} \times \boldsymbol{e}_{\theta}$ denotes the unit vector in the azimuthal direction. Defining

$$
\begin{gathered}
\boldsymbol{b}_{i l}(r, \theta)=b_{i l}^{0} \boldsymbol{\nabla} \times \boldsymbol{\nabla} \times\left\{r j_{l}\left(x_{i l} \frac{r}{r_{0}}\right) P_{l}(\cos \theta)\right\} \\
=b_{i l}^{0}\left[\boldsymbol{e}_{r} \frac{l(l+1)}{r} j_{l}\left(x_{i l} \frac{r}{r_{0}}\right) P_{l}(\cos \theta)\right. \\
\left.\quad-e_{\theta}\left\{\frac{x_{i l}}{r_{0}} j_{l}^{\prime}\left(x_{i l} \frac{r}{r_{0}}\right)+\frac{1}{r} j_{l}\left(x_{i l} \frac{r}{r_{0}}\right)\right\} P_{l}(\cos \theta) \sin \theta\right], \\
c_{l}(r, \theta)=\nabla \varphi_{l}(r, \theta)=\nabla\left\{\left(\frac{r}{r_{0}}\right)^{l} P_{l}(\cos \theta)\right\} \\
=e_{r} \frac{r^{l-1}}{r_{0}^{l}} P_{l}(\cos \theta)-e_{\theta} \frac{r^{l-1}}{r_{0}^{l}} P_{l}(\cos \theta) \sin \theta,
\end{gathered}
$$

where $j_{l}^{\prime}$ denotes the derivative of $j_{l}$, we can expand any linear droplet oscillation of the lth surface mode in the following way:

$$
\begin{gathered}
\left\{\begin{array}{c}
a_{l}(t) \\
\boldsymbol{v}_{l}(r, \theta, t)
\end{array}\right\}=\sum_{i} B_{i l}(t)\left\{\begin{array}{c}
a_{i l}^{0} \\
\boldsymbol{b}_{i l}(r, \theta)+c_{i l}^{0} c_{l}(r, \theta)
\end{array}\right\}, \\
B_{i l}(t)=B_{i l}(0) \mathrm{e}^{-\lambda_{i l} t} .
\end{gathered}
$$

Each mode

$$
\left\{\begin{array}{c}
a_{i l}^{0} \\
\boldsymbol{b}_{i l}(r, \theta)+c_{i l}^{0} c_{l}(r, \theta)
\end{array}\right\}
$$

contributes to surface deformation $\left(a_{i l}^{0}\right)$, vortex flow $\left(b_{i l}\right)$ and potential flow $\left(c_{i l}^{0} c_{l}\right)$.

\section{Mode expansion and dynamics of nonlinear droplet oscillations}

In this section we introduce a new approach to the nonlinear free boundary problem (2.1)-(2.9) which allows us to find a semi-analytical solution by mode expansion and application of the variational principle of Gauss to the hydrodynamic equations. It is based on the following premises:

(i) The boundary conditions are used either to eliminate dependent variables or become additional constraints on the vorticity equation.

(ii) The partial solutions provided by mode analysis must certainly be modified.

(iii) The linear and low viscous limits must result. 
(a)

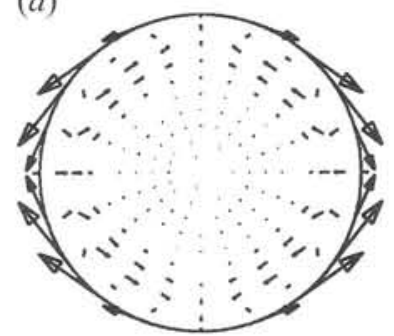

(b)

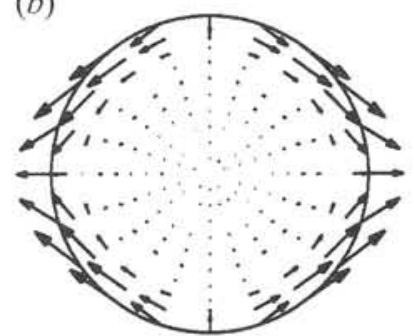

FIGURE 3. (a) Real and (b) imaginary part of the velocity field $b_{12}$ (definition (3.12)) inside an undeformed droplet. The dimensionless viscosity of $v=0.01123 r_{0}^{2} / T_{0}\left(\left|\alpha^{2}\right|=145.4\right.$ in the case of $l=2$ ) corresponds to droplets observed in our experiments.

(a)

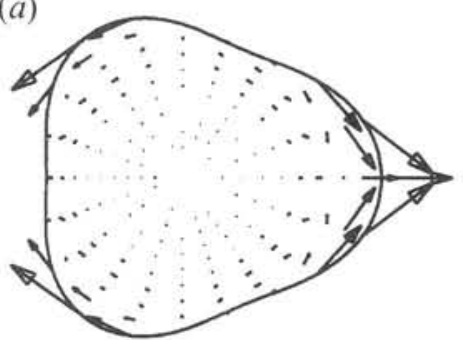

(b)

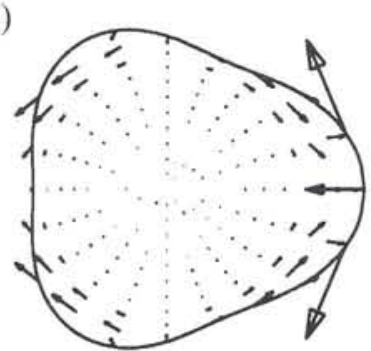

FIGURE 4. As figure 3 but within a weakly deformed droplet, and the lengthscale of the flow vectors is halved.

The surface parameterization is given by (2.1). The mode expansions of the velocity field and vorticity contain several modifications:

$$
\begin{gathered}
\boldsymbol{v}(r, \theta, t)=\sum_{l=1}^{l_{0}} \sum_{i=1}^{i_{0}} B_{i l}(t) b_{i l}\left(r, \theta ; a_{2} \ldots a_{l_{0}}\right)+\sum_{l=1}^{l_{0}} c_{l}(t) c_{l}(r, \theta), \\
w(r, \theta, t)=\sum_{l=1}^{l_{0}} \sum_{i=1}^{i_{0}} B_{i l}(t) w_{i l}\left(r, \theta ; a_{2} \ldots a_{l_{0}}\right) \boldsymbol{e}_{\phi}, \\
w_{i l}\left(r, \theta ; a_{2} \ldots a_{l_{0}}\right) \boldsymbol{e}_{\phi}=\nabla \times \boldsymbol{b}_{i l}\left(r, \theta ; a_{2} \ldots a_{l_{0}}\right) .
\end{gathered}
$$

First the fixed coupling $c_{l}=\sum B_{i l} c_{i l}^{0}$ of the potential and vortex modes is removed. In the linear analysis this coupling arises because the tangential stress of each velocity mode $b_{i l}+c_{i l}^{0} c_{l}$ vanishes at the undeformed droplet surface. Obviously this cannot hold in the case of nonlinear deformations.

Furthermore $\boldsymbol{b}_{i l}$ and $w_{i l}$ become dependent on the surface parameters. This modification generates reasonable vortex modes for arbitrary droplet shapes. The former modes (3.10) are not appropriate as the spherical Bessel functions $j_{l}$ with complex argument grow exponentially. Therefore the vorticity of the weakly damped modes is concentrated in a sheet below the droplet surface (see figure 3), and its thickness vanishes with the damping. This is a mathematical equivalent of Lamb's irrotational approximation where potential flow inside the droplet and finite vorticity at the surface are assumed. For the same reason the boundary layers of the modes (3.15) change significantly even if the deformations of the droplet are small (see figure 4).

It is expected that the boundary layers should adjust their structures to the actual droplet shape. Indeed, this is accomplished by modifying the argument of the spherical 
Bessel functions in (3.12), substituting for $r_{0}$ the time- and angle-dependent droplet radius

yielding

$$
x_{i l} \frac{r}{r_{0}} \rightarrow x_{i l} \frac{r}{R(\theta, t)},
$$

$$
\begin{gathered}
\boldsymbol{b}_{i l}\left(r, \theta ; a_{2} \ldots a_{l_{0}}\right)=b_{i l}^{0} \nabla \times\left\{j_{l}\left(x_{i l} \frac{r}{R(\theta, t)}\right) P_{l}^{\prime}(\cos \theta) \sin \theta \boldsymbol{e}_{\phi}\right\} \\
=b_{i l}^{0}\left[\boldsymbol{e}_{r}\left\{\frac{l(l+1)}{r} j_{l}\left(x_{i l} \frac{r}{R}\right) P_{l}(\cos \theta)-\frac{R_{\theta}}{R} \frac{x_{i l}}{R} j_{l}^{\prime}\left(x_{i l} \frac{r}{R}\right) P_{l}^{\prime}(\cos \theta) \sin \theta\right\}\right. \\
\left.-e_{\theta}\left\{\frac{x_{i l}}{R} j_{l}^{\prime}\left(x_{i l} \frac{r}{R}\right)+\frac{1}{r} j_{l}\left(x_{i l} \frac{r}{R}\right)\right\} P_{l}(\cos \theta) \sin \theta\right] .
\end{gathered}
$$

Figure 5 shows that the new vortex modes (4.5) behave as expected, i.e. their boundary layers agree with the droplet surface. Unfortunately the simple modification (4.4) not only results in rather complicated formulae for the derivatives of $\boldsymbol{b}_{i l}$ but it also produces singularities at the origin $O$. In particular, singularities of $w_{i l}, \boldsymbol{\nabla} \times \boldsymbol{\nabla} \times \boldsymbol{b}_{i l}$ and $\boldsymbol{\nabla} \times \boldsymbol{\nabla} \times\left\{w_{i l} \boldsymbol{e}_{\phi}\right\}$ occur for $l=1, l=1,2$ and $l=1,2,3$, respectively. This is a result of the angle-dependent scaling of the radius $r$ which produces distortions everywhere, especially close to the origin. We could avoid singularities at the expense of more complicated modifications and thus even more complicated formulae for the derivatives. Therefore we keep the scaling (4.4), trying rather to eliminate the influence of singularities on the overall solution. This is possible because the effects of vorticity are only relevant close to the surface. Hence, without losing accuracy we may split the droplet interior into two domains: a little sphere of radius $\epsilon$ surrounding the origin where we set the rotational part of the flow equal to zero, and the rest where (4.5) is valid. The thickness of the 'vortex' layer depends on the viscosity; therefore $\epsilon$ should remain small compared to $r_{0}$ in order to assure validity of the solution for highly viscous liquids. The irrotational approximation of Lamb and the nonlinear droplet model of Lundgren \& Mansour (1988) are limiting cases of this boundary-layer approximation.

The potential modes $c_{l}$ (defined in (3.13)) were used previously to describe the inviscid flow in the nonlinear case (Becker et al. 1991). They are taken without any modification.

With the mode expansion (2.1) and (4.1) defined so far the mean square errors of the governing equations (2.6)-(2.9) are defined in the following way:

$$
\begin{gathered}
\chi_{V E}^{2}=\int_{-1}^{1} \int_{e}^{R} r^{2} \mathrm{~d} r \mathrm{~d} \cos \theta\left[\sum_{i l} \dot{B}_{i l} w_{i l}+\sum_{i l} B_{i l}\left(\partial_{t} w_{i l}\right)\right. \\
\left.+\boldsymbol{e}_{\phi} \cdot(\nu \boldsymbol{\nabla} \times \boldsymbol{\nabla} \times \boldsymbol{w}-\boldsymbol{\nabla} \times(\boldsymbol{v} \times \boldsymbol{w}))\right]^{2}, \\
\chi_{K B C}^{2}=\int_{-1}^{1}\left[v \cdot\left(\boldsymbol{R} \boldsymbol{e}_{r}-\partial_{\theta} R \boldsymbol{e}_{\theta}\right)-R \partial_{t} R\right]^{2} \mathrm{~d} \cos \theta, \\
\chi_{T S C}^{2}=\frac{1}{\rho \nu} \int_{-1}^{1}[(\boldsymbol{T} n) \cdot \boldsymbol{t}]^{2} \mathrm{~d} \cos \theta, \\
\chi_{D B C}^{2}=\int_{-1}^{1}\left[\frac{1}{\rho}(\boldsymbol{T} n) \cdot \boldsymbol{n}-\frac{2 \sigma}{\rho} H\right]^{2} \mathrm{~d} \cos \theta .
\end{gathered}
$$

The problem is solved by minimizing the least-square error $\chi_{V E}^{2}$ of the vorticity equation with the constraints given by minimizing (4.8)-(4.10). 
(a)

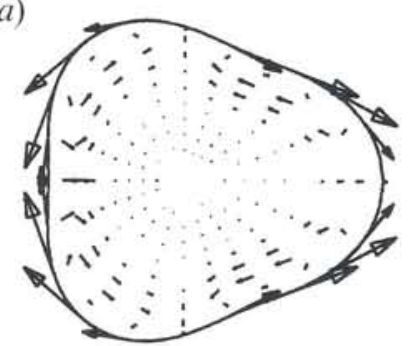

(b)

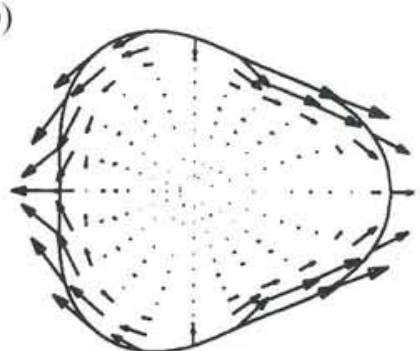

FIGURE 5. (a) Real and (b) imaginary part of $b_{12}$ (definition (4.5)) within a weakly deformed droplet, obtained with the viscosity and lengthscale of figure 3 .

It turns out to be appropriate to choose the surface parameters $a_{l}$ and the amplitude $B_{i l}$ of the vorticity as independent variables. Hence, the amplitudes $c_{l}$ of the potential flow as well as the time derivative $\dot{a}_{l}$ and $\dot{B}_{i l}$ must be found by variation.

The potential flow is determined by the tangential stress condition:

$$
\begin{aligned}
\chi_{T S C}^{2}= & \int \mathrm{d} \cos \theta\left[\sum_{l=1}^{l_{0}} \sum_{i=1}^{i_{0}} B_{i l}\left\{2(\boldsymbol{n} \cdot \boldsymbol{\nabla}) \boldsymbol{b}_{i l}+\boldsymbol{n} \times \boldsymbol{\nabla} \times \boldsymbol{b}_{i l}\right\} \cdot \boldsymbol{t}\right. \\
& \left.+\sum_{l=2}^{l_{0}} c_{l}\left\{2(\boldsymbol{n} \cdot \boldsymbol{\nabla}) \boldsymbol{c}_{l}\right\} \cdot \boldsymbol{t}\right]^{2} \rightarrow \min \\
\Rightarrow & \partial \chi_{T S C}^{2} / \partial c_{l}=0, \quad l=2 \ldots l_{0} .
\end{aligned}
$$

The amplitude $c_{1}$ cannot be evaluated from (4.11) because the tangential stress of the homogeneous flow $c_{l=1}$ vanishes. We proceed, eliminating $c_{1}$ and the surface velocities $\dot{a}_{l}$ from the kinematic boundary condition:

$$
\begin{aligned}
\chi_{K B C}^{2}= & \int \mathrm{d} \cos \theta\left[c_{1} c_{1} \cdot\left(R \boldsymbol{e}_{r}-R_{\theta} \boldsymbol{e}_{\theta}\right)-\sum_{l=2}^{l_{0}} \dot{a}_{l} R \frac{\partial R}{\partial a_{l}}\right. \\
& \left.+\left(\sum_{l=2}^{l_{0}} c_{l} c_{l}+\sum_{l=1}^{l_{0}} \sum_{i=1}^{i_{0}} B_{i l} \boldsymbol{b}_{i l}\right) \cdot\left(R \boldsymbol{e}_{r}-R_{\theta} \boldsymbol{e}_{\theta}\right)\right]^{2} \rightarrow \min \\
\Rightarrow & \partial \chi_{K B C}^{2} / \partial c_{1}=0, \quad \partial \chi_{K B C}^{2} / \partial \dot{a}_{l}=0, \quad l=2 \ldots l_{0} .
\end{aligned}
$$

The results of (4.11) and (4.12) can formally be written as

$$
\begin{aligned}
c_{l} & =\sum_{m=1}^{l_{0}} \sum_{i=1}^{i_{0}} C_{i m}^{l}\left(a_{2} \ldots a_{l_{0}}\right) B_{i m}, \quad l=1 \ldots l_{0}, \\
\dot{a}_{l} & =\sum_{m=1}^{l_{0}} \sum_{i=1}^{i_{0}} K_{i m}^{l}\left(a_{2} \ldots a_{l_{0}}\right) B_{i m}, \quad l=2 \ldots l_{0}, \\
-\dot{s} & =-\sum_{l=2}^{l_{0}} \frac{\partial s}{\partial a_{l}} \dot{a}_{l}=-\sum_{m=1}^{l_{0}} \sum_{i=1}^{i_{0}} S_{i m}\left(a_{2} \ldots a_{l_{0}}\right) B_{i m} .
\end{aligned}
$$

So far all expansion parameters for velocities and the first differential equations, namely (4.14) are known.

The accelerations, i.e. differential equations of the form $\dot{B}_{i l}=\ldots$ must be derived from the dynamic boundary condition (2.9) and the vorticity equation (2.6) in one step. These equations are fundamentally of differing importance to the problem. Whereas the vorticity equation plays its role only for viscous flow, the driving force for oscillations - the surface tension - dominates droplet dynamics in general, independent 
of the presence of viscous effects. Therefore it is most important to satisfy the dynamic boundary condition with the smallest possible error. Hence we must determine the unknown time derivatives $\dot{B}_{i l}$ by minimizing the mean error $\chi_{D B C}^{2}$ without any regard to the value of $\chi_{V E}^{2}$. The remaining flexibility of the modes must be used to satisfy the vorticity equation.

Accordingly we rewrite the dynamic boundary condition (2.9), substituting the pressure by line integration of the Navier-Stokes equation (2.5):

$$
\int_{0}^{R}\left(\partial_{t} v-\ddot{s} \boldsymbol{e}_{z}+(\boldsymbol{v} \cdot \boldsymbol{\nabla}) v+\nu \nabla \times \nabla \times v\right) \cdot \mathrm{d} \boldsymbol{r}+\dot{c}_{0}+\nu\{2(\boldsymbol{n} \cdot \boldsymbol{\nabla}) \boldsymbol{v}\} \cdot \boldsymbol{n}=\frac{2 \sigma}{\rho} H ;
$$

$\dot{c}_{0}$ is a time-dependent constant of integration and equal to $-2 \sigma / \rho r_{0}$ in the linear limit. By inserting expansion (4.1) into (4.16), the mean-square error (4.10) is transformed to

$$
\begin{aligned}
\chi_{D B C}^{2}= & \int_{-1}^{1} \mathrm{~d} \cos \theta\left[\sum_{l=1}^{l_{0}} \sum_{i=1}^{i_{0}} \partial_{t}\left\{B_{i l} \int_{\epsilon}^{R} \boldsymbol{b}_{i l} \cdot \mathrm{d} \boldsymbol{r}\right\}+\sum_{l=1}^{l_{0}} \dot{c}_{l} \varphi_{l}-\ddot{s} \varphi_{1}+\frac{1}{2} \boldsymbol{v}^{2}\right. \\
& \left.-\int_{\epsilon}^{R}(\boldsymbol{v} \times \boldsymbol{w}-\nu \boldsymbol{\nabla} \times \boldsymbol{\nabla} \times \boldsymbol{v}) \cdot \mathrm{d} \boldsymbol{r}+\nu\{2(\boldsymbol{n} \cdot \boldsymbol{\nabla}) \boldsymbol{v}\} \cdot \boldsymbol{n}-\frac{2 \sigma}{\rho} H\right]^{2} \\
= & \int_{-1}^{1} \mathrm{~d} \cos \theta F_{D B C}^{2} .
\end{aligned}
$$

In (4.17) the lower integration limit $r=0$ has been replaced by $\epsilon$ in order to separate the singularities of the rotational modes (4.5). $F_{D B C}$ is the local error of the dynamic boundary condition.

If viscous effects were absent, the flow would be described by the velocity potential $\Sigma_{c_{l} \varphi_{l}}$ alone. In accordance with the boundary-layer argument, the bulk flow can be considered as nearly irrotational in the case of damped oscillations also. Hence, $\chi_{D B C}^{2}$ become small if we choose formally the time derivatives $\dot{c}_{l}$ as variational parameters. From (4.17) we see that this variation is equivalent to the projection of $F_{D B C}$ onto the potentials $\varphi_{l}$. Thus we obtain the following set of $l_{0}+1$ independent equations from the dynamic boundary condition:

$$
h_{l}=\partial \chi_{D B C}^{2} / \partial \dot{c}_{l}=\int_{-1}^{1} \mathrm{~d} \cos \theta F_{D B C} \varphi_{l}=0, \quad l=0 \ldots l_{0} .
$$

Of course, these additional constraints are calculated after inserting (4.13) into $F_{D B C}$ :

$$
\begin{aligned}
F_{D B C}= & \sum_{l=1}^{l_{0}} \sum_{i=1}^{i_{0}} \dot{B}_{i l}\left(\int_{\epsilon}^{R} b_{i l} \cdot \mathrm{d} \boldsymbol{r}+\sum_{n=1}^{l_{0}} C_{i l}^{n} \varphi_{n}-S_{i l} \varphi_{1}\right)+\dot{c}_{0} \varphi_{0} \\
& +\sum_{l=1}^{l_{0}} \sum_{i=1}^{i_{0}} B_{i l} \int_{\epsilon}^{R}\left(\partial_{t} \boldsymbol{b}_{i l}\right) \cdot \mathrm{d} \boldsymbol{r}+\sum_{l=2}^{l_{0}}\left(\sum_{n=1}^{l_{0}} \frac{\partial c_{n}}{\partial a_{l}} \varphi_{n}-\frac{\partial \dot{S}}{\partial a_{l}} \varphi_{1}\right) \dot{a}_{l} \\
& +\frac{1}{2} v^{2}+\int_{\epsilon}^{R}(\nu \nabla \times \nabla \times v-v \times w) \cdot \mathrm{d} \boldsymbol{r}-v\{2(\boldsymbol{n} \cdot \boldsymbol{\nabla}) \boldsymbol{v}\} \cdot \boldsymbol{n}-\frac{2 \sigma}{\rho} H .
\end{aligned}
$$

Now the outstanding differential equations result simply from the variation $\chi_{V E}^{2} \rightarrow \min$ with the constraints (4.18):

$$
\left.\begin{array}{rl}
h_{l} & =0, \quad l=0 \ldots l_{0} ; \\
\frac{\partial \chi_{V E}^{2}}{\partial \dot{B}_{i l}}+\sum_{m=0}^{l_{0}} \kappa_{m} \frac{\partial h_{m}}{\partial \dot{B}_{i l}} & =0, \quad i=1 \ldots i_{0}, \quad l=1 \ldots l_{0} ; \\
\frac{\partial \chi_{V E}^{2}}{\partial \dot{c}_{0}}+\sum_{m=0}^{l_{0}} \kappa_{m} \frac{\partial h_{m}}{\partial \dot{c}_{0}} & =0 .
\end{array}\right\}
$$


The vorticity equation and dynamic boundary equation are weighted automatically because the set of equations (4.20) determines not only $\dot{B}_{i l}$ and $\dot{c}_{0}$ but also the Lagrange multipliers $\kappa_{m}$. In the linear limit they become equal to zero. The constraint (4.18), appearing as the first $\left(l_{0}+1\right)$ equations of (4.20), ensure the correct solution for the low viscous limit.

Owing to the complex roots $x_{i l}$ of the characteristic equation (3.13) the parameters determined by the minimization procedure are generally complex numbers. To find real modes and real amplitudes every expansion containing the weakly damped modes or their derivatives must be rearranged to separate their real and imaginary parts. For simplicity our notation describes full complex functions.

The prime errors (4.7)-(4.10) are normalized by referring them to the mean-square values of the corresponding functions that are approximated by the mode expansions:

$$
\begin{gathered}
\chi_{V E-r e l}^{2}=\chi_{V E}^{2} / \int_{-1}^{1} \int_{\epsilon}^{R} r^{2} \mathrm{~d} r \mathrm{~d} \cos \theta\left[\sum_{l=1}^{l_{0}} \sum_{i=1}^{i_{0}} B_{i l}\left(\partial_{t} w_{i l}\right)+\boldsymbol{e}_{\phi} \cdot(\nu \boldsymbol{\nabla} \times \boldsymbol{\nabla} \times \boldsymbol{w}-\boldsymbol{\nabla} \times(\boldsymbol{v} \times \boldsymbol{w}))\right]^{2}, \\
\chi_{K B C-r e l}^{2}=\chi_{K B C}^{2} / \int_{-1}^{1} \mathrm{~d} \cos \theta\left[\left(\sum_{l=1}^{l_{0}} \sum_{i=1}^{i_{0}} B_{i l} \boldsymbol{b}_{i l}+\sum_{l=2}^{l_{0}} c_{l} c_{l}\right) \cdot\left(R \boldsymbol{e}_{r}-R_{\theta} \boldsymbol{e}_{\theta}\right)\right]^{2}, \\
\chi_{T S C-r e l}^{2}=\chi_{T S C}^{2} / \int_{-1}^{1} \mathrm{~d} \cos \theta\left[\sum_{l=1}^{l_{0}} \sum_{i=1}^{i_{0}} B_{i l}\left\{2(\boldsymbol{n} \cdot \boldsymbol{\nabla}) \boldsymbol{b}_{i l}+\boldsymbol{n} \times \boldsymbol{\nabla} \times \boldsymbol{b}_{i l}\right\} \cdot \boldsymbol{t}\right]^{2}, \\
\chi_{D B C-r e l}^{2}=\chi_{D B C}^{2} / \int_{-1}^{1} \mathrm{~d} \cos \theta\left[\sum_{l=1}^{l_{0}} \sum_{i=1}^{i_{0}} B_{i l} \int_{e}^{R}\left(\partial_{t} \boldsymbol{b}_{i l}\right) \cdot \mathrm{d} \boldsymbol{r}+\sum_{l=2}^{l_{0}}\left(\sum_{n=1}^{l_{0}} \frac{\partial c_{n}}{\partial a_{l}} \varphi_{n}-\frac{\partial \dot{s}}{\partial a_{l}} \varphi_{1}\right) \dot{a}_{l}\right. \\
\left.+\frac{1}{2} v^{2}+\int_{\epsilon}^{R}(\nu \nabla \times \nabla \times \boldsymbol{v}-\boldsymbol{v} \times \boldsymbol{w}) \cdot \mathrm{d} \boldsymbol{r}-v\{2(\boldsymbol{n} \cdot \boldsymbol{\nabla}) \boldsymbol{v}\} \cdot \boldsymbol{n}-\frac{2 \sigma}{\rho} H\right]^{2} .
\end{gathered}
$$

These relations are used to measure the systematic errors of the governing equations (2.6)-(2.9).

The final differential equations (4.14) and (4.20) are solved numerically applying the following standard methods:

(i) extrapolation in rational functions and polynomial extrapolation to evaluate integrals and derivatives (Stoer 1972);

(ii) Gauss elimination to solve systems of algebraic equations;

(iii) a modified Runge-Kutta algorithm (Fehlberg 1970) to integrate ordinary differential equations; and

(iv) approximation of the spherical Bessel functions by Legendre polynomials to reduce computational time (Amos 1986).

Finally let us consider the linear limit of the model. We have chosen the surface coefficients $a_{l}$ and the vorticity amplitudes $B_{i l}$ a degrees of freedom. Therefore one might expect inconsistency with the linear theory in which only the $B_{i l}$ are independent parameters and the $a_{l}$ are always given by

$$
\sum_{i=1}^{i_{0}} B_{i l} a_{i l}^{0}-a_{l}=0, \quad l=2 \ldots l_{0}
$$

(see (3.14)). This problem would be avoided if the fixed relations (4.25) and their time derivatives were considered as additional constraints. However, a greater flexibility of the mode expansions seems preferable to us. The example shown in figure 6 confirms 

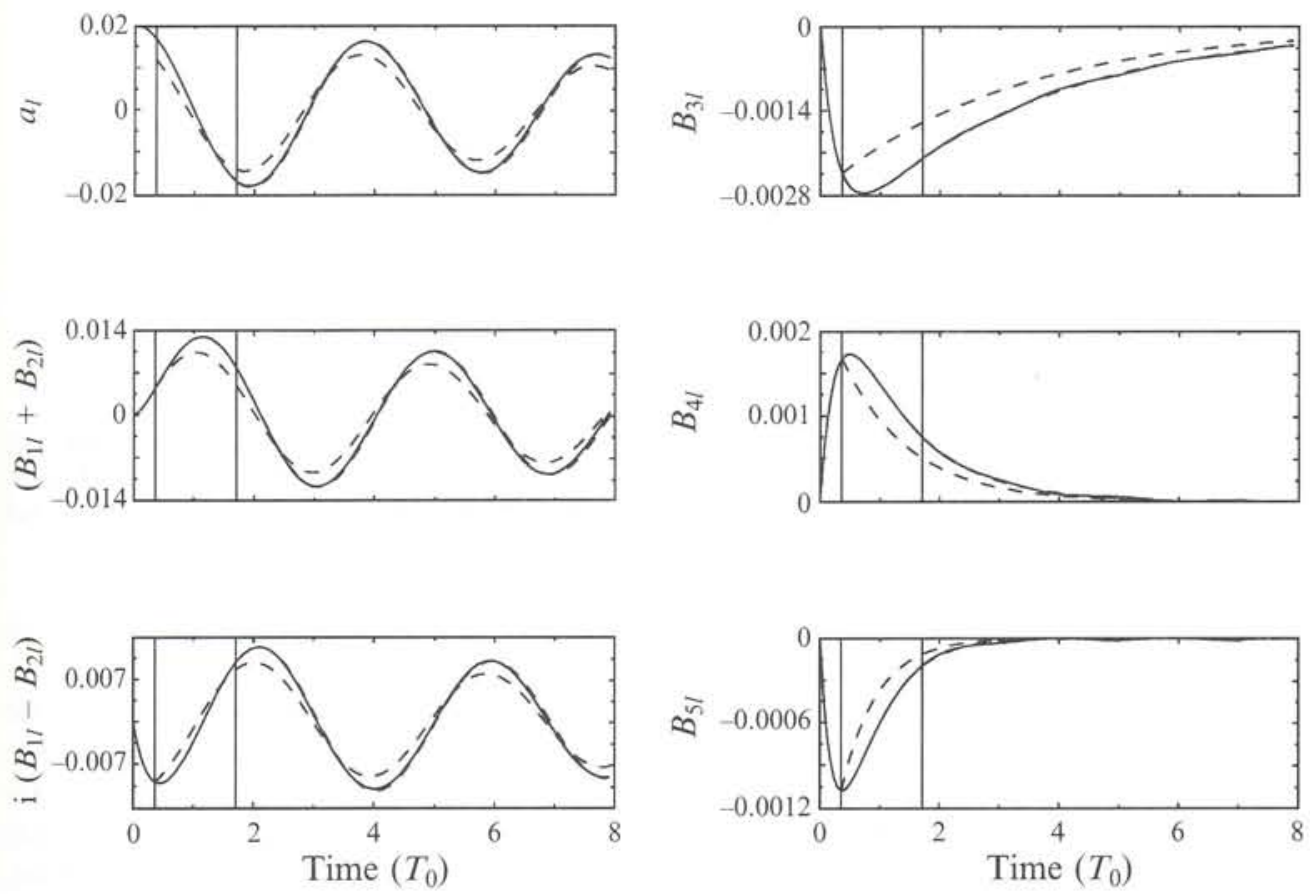

Figure 6. Computation in the linear limit. Truncation numbers and initial conditions are $i_{0}=5$, $I_{0}=2, a_{2}(0)=0.02$ and $B_{i l}(0)=0$. The radius of the irrotational region $\epsilon=0.1 r_{0}$, and the dimensionless viscosity $\nu=0.00123 r_{0}^{2} / T_{0}$. The solid curves show the results of the nonlinear model. The dashed curves follow from linear theory (see (3.14)). They start at $t=0.36 T_{0}$ and $t=1.17 T_{0}$ indicated by solid vertical lines. Their initial conditions are the actual values $B_{i l}(t)$ of the nonlinear numerical solution.

that the present model behaves correctly in the linear limit. The computation (solid lines) begins at initial conditions not allowed by (4.25). Comparisons with the linear solutions (dashed lines) following from (3.14) show initially some deviations. However, as can be seen, these deviations disappear with time, i.e. the nonlinear model approaches the linear solutions.

\section{Results}

\subsection{Initial conditions}

Our experimental method of generating and evaluating oscillating droplets has already been described in recently published papers (Hiller \& Kowalewski 1989; Becker et al. 1991). Strongly deformed axisymmetric droplets of about $0.5 \mathrm{~mm}$ in diameter are produced by the controlled breakup of a liquid jet, and the time evolution of a droplet cross-section is observed using a stroboscopic illumination technique. Further analysis consists of fitting the function (2.1) with a truncation number $l_{0}=5$ to the recorded droplet shapes. This yields the surface parameters $a_{2} \ldots a_{5}$ and the equivolumetric radius $r_{0}$ as functions of time. Usually the droplet radius decreases weakly with time owing to evaporation. In the present model we neglect this small effect and assume for $r_{0}$ its mean experimental value.

In the following we compare two typical experimental results already given in Becker et al. (1991, figures 5 and 6) with the theoretical predictions. Figure 7 shows an example with large initial amplitudes. 

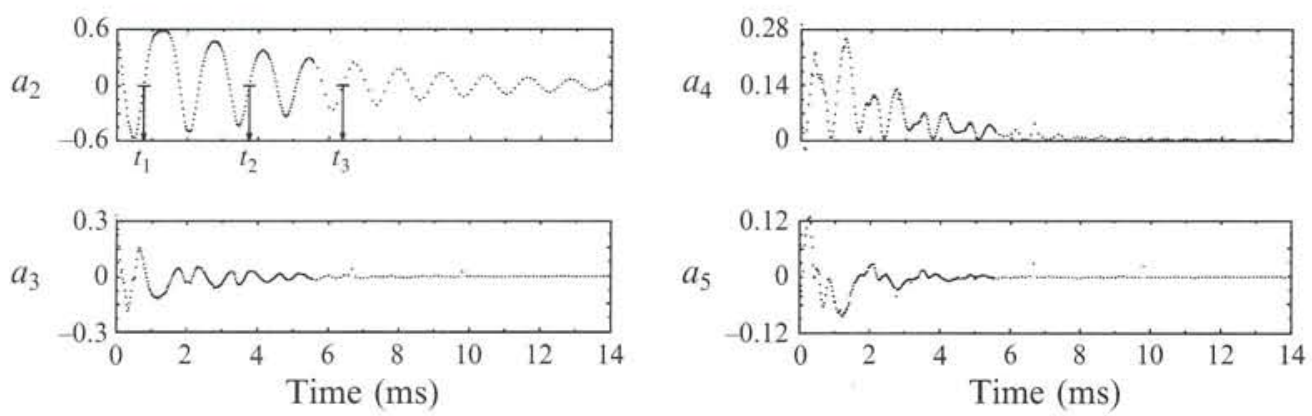

FIGURE 7. Experimental result for an ethanol droplet oscillating in air. The surface parameters (dotted lines) $a_{2} \ldots a_{5}$ are shown as functions of time. Each dot corresponds to each time the droplet cross-section was recorded and analysed. The mean equivolumetric radius of this measurement was $r_{0}=207 \mu \mathrm{m} . t_{1}, t_{2}$ and $t_{3}$ mark points in time where computations with the model were started.

The flow field inside the droplet cannot be determined experimentally. Observations of droplets yield solely the surface parameters $a_{l}$ and eventually their time derivatives $\dot{a}_{l}$ (which can be evaluated by interpolating $a_{l}(t)$ ). Therefore, it is difficult to formulate exact adequate initial conditions for the model. However, the $a_{l}$ and $\dot{a}_{l}$ contain more information than one might expect. With the help of two additional assumptions it is possible to compute reasonable initial amplitudes $B_{i l}$ from the available experimental data. These assumptions, justified by comparison of numerical and experimental data, are: (i) the strongly dissipative modes can be neglected, and (ii) the amplitudes obey the relations (4.25). These postulates correspond to the irrotational case where the velocity field is determined by the surface motion only. Accordingly the tangential stress conditions, (4.11) yields

$$
c_{l}=\sum_{m=2}^{l_{0}} \sum_{i=1}^{2} C_{i m}^{l}\left(a_{2} \ldots a_{l_{0}}\right) B_{i m}, \quad l=2 \ldots l_{0},
$$

and the kinematic boundary condition (4.12) can be rewritten in the form

$$
\begin{aligned}
& \chi_{K B C}^{2}=\int \mathrm{d} \cos \theta\left[\left(c_{1} c_{1}+\sum_{l=2}^{l_{0}} \sum_{i=1}^{2} B_{i l}\left(\boldsymbol{b}_{i l}+\sum_{m=2}^{l_{0}} C_{i l}^{m} \boldsymbol{c}_{m}\right)\right) \cdot\left(\boldsymbol{R} \boldsymbol{e}_{r}-\partial_{\theta} R \boldsymbol{e}_{\theta}\right)\right. \\
&\left.+R \sum_{l=2}^{l_{0}} \frac{\partial R}{\partial a_{l}} \dot{a}_{l}\right]^{2} \rightarrow \text { min. }
\end{aligned}
$$

The couplings (4.25) now become additional constraints on (5.2) because variation of $\chi_{K B C}^{2}$ with respect to $c_{1}$ and $B_{i 1}$ gives only $l_{0}$ independent equations. Analogously to (4.20) we obtain the following system of algebraic equations:

$$
\left.\begin{array}{l}
K_{l}=a_{l}-\sum_{l=2}^{l_{0}} \sum_{i=1}^{2} a_{i l}^{0} B_{i l}=0, \quad l=2 \ldots l_{0}, \\
\frac{\partial \chi_{K B C}^{2}}{\partial c_{1}}+\sum_{m=2}^{l_{0}} \kappa_{m} \frac{\partial K_{m}}{\partial c_{1}}=0, \\
\frac{\partial \chi_{K B C}^{2}}{\partial B_{i l}}+\sum_{m=2}^{l_{0}} \kappa_{m} \frac{\partial K_{m}}{\partial B_{i l}}=0, \quad i=1,2, \quad l=2 \ldots l_{0} .
\end{array}\right\}
$$

All our nonlinear computations presented in the following sections were started using (5.3) to calculate initial conditions for the new model from the initial values of the surface parameters $a_{l}$ and their velocities $\dot{a}_{l}$. 

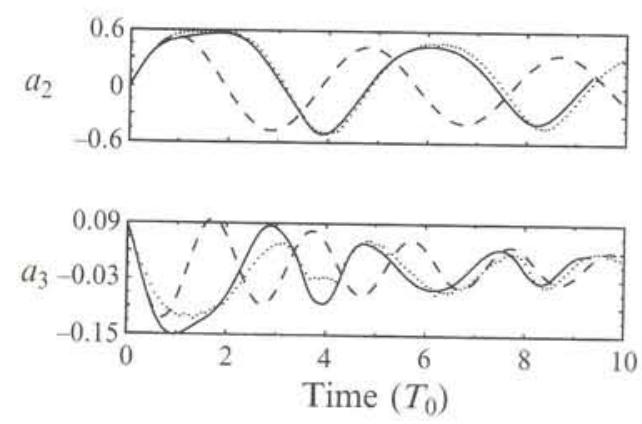
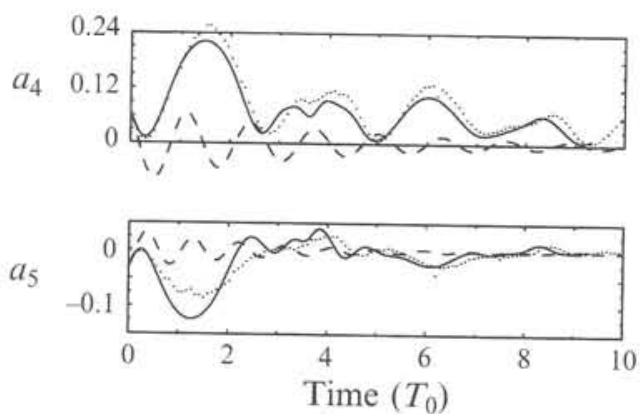

FigURE 8. Computational results (solid lines) starting at $t_{1}$ (cf. figure 7). In addition the experimental data (dotted lines) and the predictions of linear theory (dashed lines) are included. The initial conditions and model parameters are $a_{2}=0, a_{3}=0.086, a_{4}=0.065, a_{5}=-0.03, \dot{a}_{2}=0.9 / T$ $\dot{a}_{3}=-0.31 / T_{0}, \dot{a}_{4}=-0.31 / T_{0}, \dot{a}_{5}=0.18 / T_{0}, \nu=0.01123 r_{0}^{2} / T_{0}, i_{0}=3, l_{0}=6$ and $\epsilon=0.1 r_{0}$.

\subsection{Comparison with experiments}

In figure $7 t_{1}, t_{2}$ and $t_{3}$ mark three zero crossings of $a_{2}(t)$, separated in each case by two oscillation periods. The experimental data at these points are used as initial conditions to start the calculation for the next interval. Hence, simulation of the whole experimental run is done in three steps, covering three regions of droplet oscillations: strong nonlinear $\left(\max \left|a_{2}(t)\right| \approx 0.6\right)$, nonlinear $\left(\max \left|a_{2}(t)\right| \approx 0.4\right)$, and quasi-linear $\left(\max \left|a_{2}(t)\right| \approx 0.2\right)$.

Calculations were performed using the physical data of the liquid used in the experiment, i.e. $\rho=803 \mathrm{~kg} / \mathrm{m}^{3}, \sigma=22.9 \times 10^{-3} \mathrm{~N} / \mathrm{m}, \nu=1.49 \times 10^{-6} \mathrm{~m}^{2} / \mathrm{s}$, respectively. The free parameters of the nonlinear model are chosen to be $i_{0}=3, l_{0}=6$ and $\epsilon=0.1 r_{0}$. Although the large-amplitude oscillations were sometimes analysed experimentally with $l_{0}=10$, amplitudes found for $l>5$ are too small to be evaluated with reliable accuracy. The initial values of $a_{6}$ and $\dot{a}_{6}$ have been set to zero as they are also not significant for the overall accuracy of the numerical analysis.

Figure 8 shows results of the first computation starting at $t_{1}$. They are compared with the experimental data and results of the linear model

$$
a_{l}(t)=\exp \left\{-\operatorname{Re}\left(\lambda_{1 l}\right) t\right\}\left(A_{1 l} \cos \left\{\operatorname{Im}\left(\lambda_{1 l}\right) t\right\}+A_{2 l} \sin \left\{\operatorname{Im}\left(\lambda_{1 l}\right) t\right\}\right),
$$

where the constants $A_{1 l}$ and $A_{2 l}$ correspond to the initial values of $a_{l}$ and $\dot{a}_{l}$.

The strongest nonlinearities are visible shortly after the droplet is generated at the tip of the jet. The maximum droplet deformation, i.e. the maximum value of $\left|\Sigma a_{l}\right|$, is approximately 0.8 . One can see that, unlike the case of the linear theory, the present model describes the experimental data very well. The following effects of nonlinearities can be readily seen, when comparing with the linear model:

(a) The oscillation period of the fundamental surface mode $a_{2}$ increases. The surface displacements are asymmetrical: the maxima (prolate deformation) are larger and flatter, whereas minima are sharper and have a smaller value. These effects are also typical for inviscid nonlinear models (Tsamopoulos \& Brown 1983).

(b) The observed nonlinearity of the first higher-order mode $a_{3}$ is even stronger; it oscillates faster for negative displacements of $a_{2}$ and clearly slower for positive ones. The combined action of $a_{2}$ and $a_{3}$ shows that the average deformation of the droplet changes faster when the droplet has an oblate shape and slower when it is elongated. These periodic frequency modulations can be understood in terms of effective masses. In inviscid theory the kinetic energy of the droplet can be written as $\frac{1}{2} \Sigma M_{l m} \dot{a}_{l} \dot{a}_{m}$. 

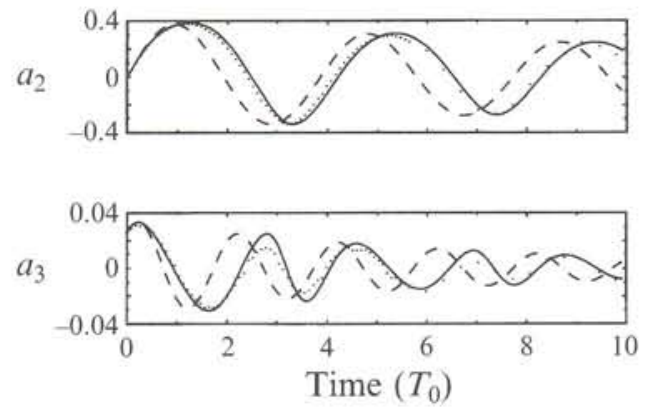
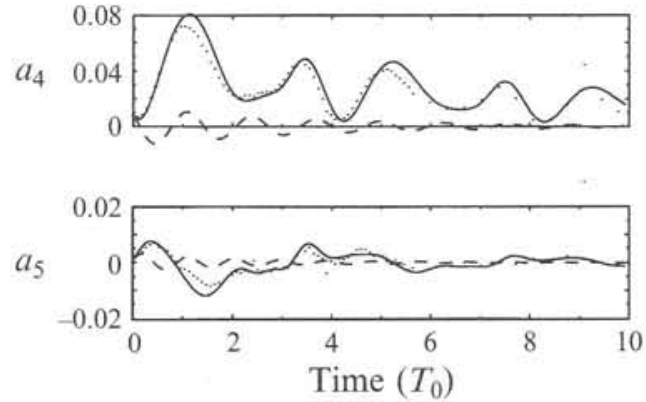

FIGURE 9. Computational results (solid lines) starting at $t_{2}$ (cf. figure 7). In addition the experimental data (dotted lines) and the predictions of linear theory (dashed lines) are included. The initial conditions are $a_{2}=0, a_{3}=0.026, a_{4}=0.009, a_{5}=0.001, \dot{a}_{2}=0.65 / T_{0}, \dot{a}_{3}=0.069 / T_{0}$, $\dot{a}_{4}=-0.058 / T_{0}, \dot{a}_{5}=0.022 / T_{0}$. The model parameters $\nu, i_{0}, l_{0}, \epsilon$ are those of figure 8 .
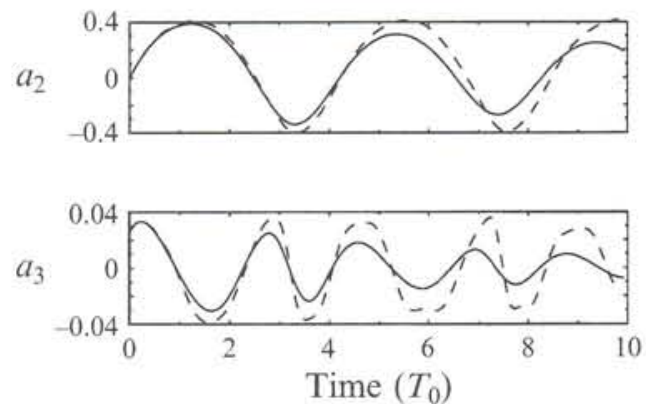
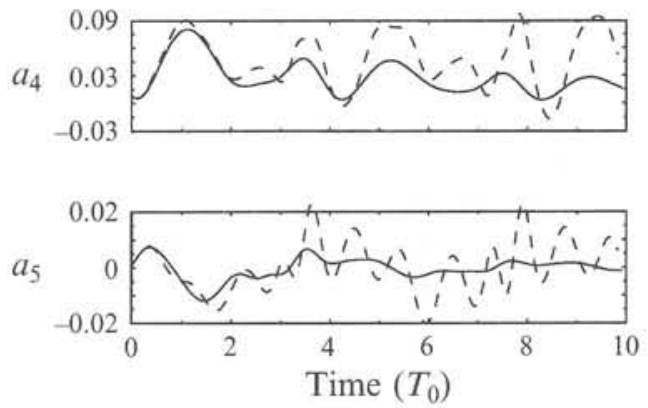

Figure 10. Computational results of the nonlinear viscous model (solid lines) taken from figure 9 compared with a corresponding inviscid calculation (dashed lines). The solution of the inviscid model (Becker et al. 1991) has been evaluated with the maximum polar wavenumber $l=6$ in the surface parametrization and velocity potential expansion.

Each diagonal element of the mass tensor $M_{l m}$ gives the inertia of a single surface wave and the non-diagonal elements describe the nonlinear couplings. Linearization leads to a diagonal matrix and effective masses $M_{l m} \propto \delta_{l m} / l(2 l+1)$. In the nonlinear case the matrix elements depend on the instantaneous droplet shape and it can be shown that a shape elongation yields growth of its diagonal elements.

(c) The third higher oscillation mode $a_{4}$ shows a strong coupling with $a_{2}$. Except for the small ripple at $t \approx 3 T_{0}$, the maxima of $a_{4}$ coincide quite well with the extremes of $a_{2}$. According to the linear analysis (compare (3.7)), one might rather expect the frequency ratio $1: 3$ instead of the observed ratio $1: 2$ and an arbitrary phase shift between both amplitudes. This indicates that predictions of the linear theory cannot be related to the real behaviour of $a_{4}$. This holds also for amplitude $a_{5}$ although the mode coupling is not so straightforward.

The predictions of the nonlinear model for the second interval $t_{2}-t_{3}$ are shown in figure 9. Comparing it with the previous interval (figure 8), one can see that the quantitative coincidence with the experimental data is clearly improved although the nonlinear characteristics remain. The short-time behaviour of the oscillation amplitudes can also be relatively well described by the former inviscid nonlinear model (figure 10).

The results of the third computational run which starts at $t_{3}$ are displayed in figure 11 together with both previous computations. They are compared with the measured 

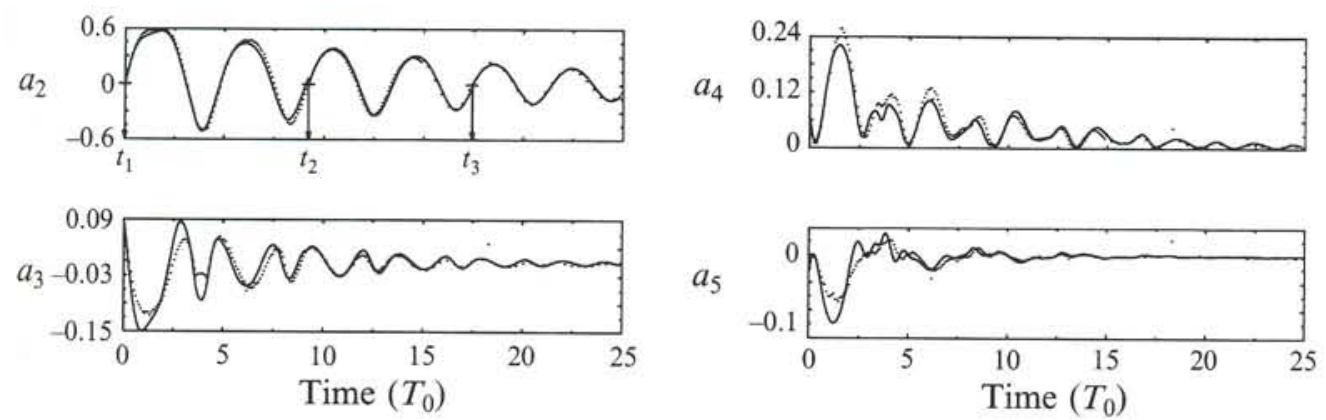

FIGURE 11. Three successive computations (solid lines) starting at $t_{1}, t_{2}$ and $t_{3}$ (cf. figure 7), compared with the experiment (dotted lines). The discontinuity of the theoretical curves at $t=t_{2}$ and $t=t_{3}$ is due to the restarting of calculations. The initial conditions for $t_{3}$ are $a_{2}=0, a_{3}=0.0003, a_{4}=0.0051$, $a_{5}=0.001, \dot{a}_{2}=0.384 / T_{0}, \dot{a}_{3}=0.029 / T_{0}, \dot{a}_{4}=-0.0034 / T_{0}, \dot{a}_{5}=-0.0031 / T_{0}$. The model parameters $v, i_{0}, l_{0}, \epsilon$ are those of figure 8 .
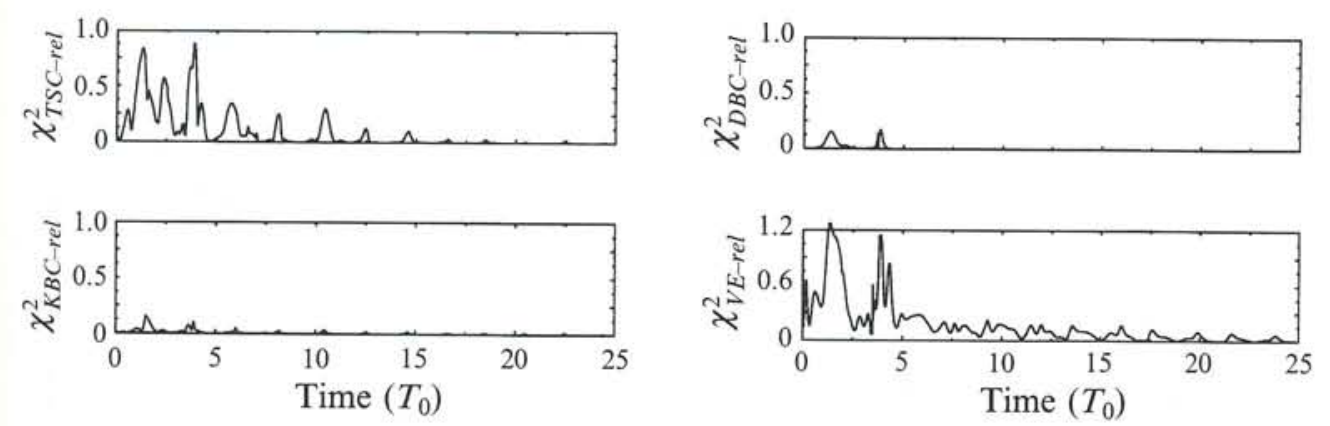

FIGURE 12. Relative mean errors of the tangential stress conditions $\left(\chi_{T S C-r e t}^{2}\right)$, kinematic boundary condition $\left(\chi_{K B C-r e l}^{2}\right)$, dynamic boundary condition $\left(\chi_{D B C-\text { rel }}^{2}\right)$ and vorticity equation $\left(\chi_{V B-\text { rel }}^{2}\right)$ (see definitions (4.21)-(4.24)) corresponding to the oscillation shown in figure 11 .

oscillation amplitudes. It can be seen that the long-time behaviour of $a_{2}$ and $a_{3}$ shows oscillations typical of the damped harmonic oscillator. Asymptotically both $a_{2}$ and $a_{3}$ approach the predictions of the linear theory. Surprisingly, even in this last-analysed interval the amplitude $a_{4}$ remains always positive, indicating the presence of the nonlinear mode coupling with $a_{2}$. The surface wave $a_{6}$ is not displayed as its small amplitudes are not of interest.

Figure 12 shows the corresponding systematic errors (4.21)-(4.24) for the three calculation runs. If strong nonlinearities are present, the tangential stress condition and vorticity equation cannot be solved appropriately. The maximum relative mean errors of these equations are of the order of $100 \%$. Nevertheless, kinematic and dynamic boundary conditions, which determine the droplet dynamics, are always solved with negligible errors and the results are in accordance with the experiment. This validates our approximation of treating the boundary conditions as additional constraints on the equation of motion. The kinematic and especially the dynamic boundary conditions are essential for the irrotational inviscid droplet dynamics. They remain dominant in the viscous case also. The tangential stress condition and the vorticity equation seem to play a secondary role, mainly affecting the amplitudes by damping.

One example of modelling another experimental run, characterized by moderate excitation amplitudes, is shown in figure 13 (compare also figure 4 in Becker et al. 

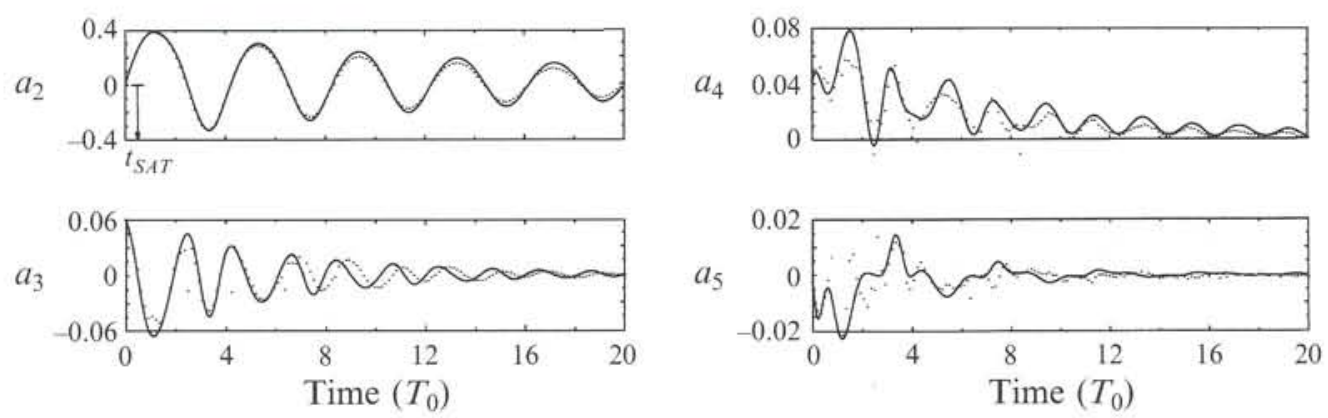

FIGURE 13. Experimental (dotted lines) and theoretical (solid lines) results of an ethanol droplet oscillating at a moderate excitation amplitude. The initial conditions are: $a_{2}=0, a_{3}=0.061$, $a_{4}=0.042, \quad a_{5}=-0.002, \quad \dot{a}_{2}=0.625 / T_{0}, \quad \dot{a}_{3}=-0.065 / T_{0}, \quad \dot{a}_{4}=0.085 / T_{0}, \quad \dot{a}_{5}=-0.103 / T_{0} . \quad$ The mean equivolumetric radius of the droplet $r_{0}=0.1733 \mathrm{~mm}$ results in a dimensionless viscosity of $\nu=0.01227 r_{0}^{2} / T_{0}$. The other parameters are $i_{0}=3, l_{0}=6$ and $\epsilon=0.1 r_{0}$. At $t=t_{S_{A T}}$ the observed droplet merges with a satellite.
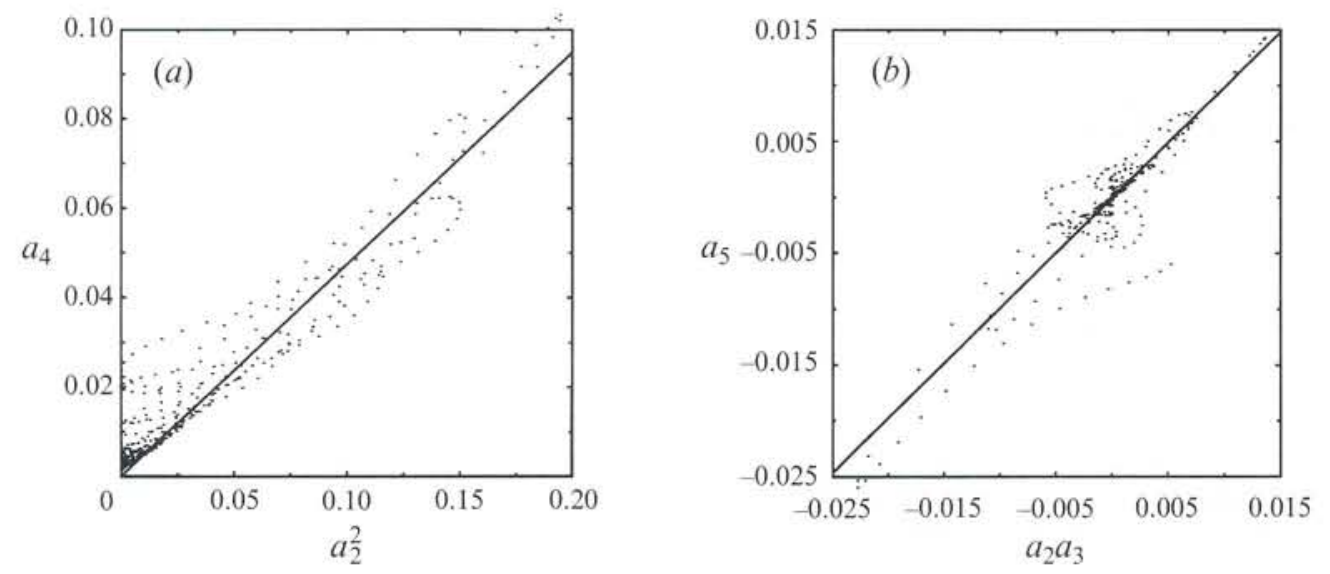

FIGURE 14. Phase-space relations of mode couplings: $(a)$ between $a_{4}$ and $a_{2}^{2} ;(b)$ between $a_{5}$ and $a_{2} a_{3}$. The dots represent numerical results obtained with the present model, taken from the data set of figure 11 for $t>5 T_{0}$. The slopes of the fitted straight lines are (a) 0.47 and (b) 0.99 .

1991). At $t=t_{S A T} \approx 0.5 T_{0}$ the observed droplet merges with a satellite droplet. It is interesting that this relatively violent disturbance has an appreciable influence only on the higher surface waves $a_{4}$ and $a_{5}$. However, as time passes, the severe deviations from the model predictions for these two amplitudes diminish. Finally the experimental and theoretical data coincide again, at least qualitatively. This leads to the assumption that $a_{4}$ and $a_{5}$ are regenerated by nonlinear interactions with $a_{2}$ and $a_{3}$ and that their longtime behaviour is independent of initial disturbances.

\subsection{Mode couplings}

The mode interaction for $a_{4}$ and $a_{5}$ with initial conditions taken from the experiments can be simply analysed in the phase space. These phase-space investigations have been done using a multi-parameter editor Relation (Wilkening 1992). In particular, reasonable results seem to be offered by the relations $a_{4}=a_{4}\left(a_{2}^{2}\right)$ and $a_{5}=a_{5}\left(a_{2} a_{3}\right)$ shown in figure 14. According to these representations the couplings of $a_{4}$ and $a_{5}$ can be approximately described by

$$
a_{4} \approx C_{1} a_{2}^{2} \text { and } a_{5} \approx C_{2} a_{2} a_{3} .
$$



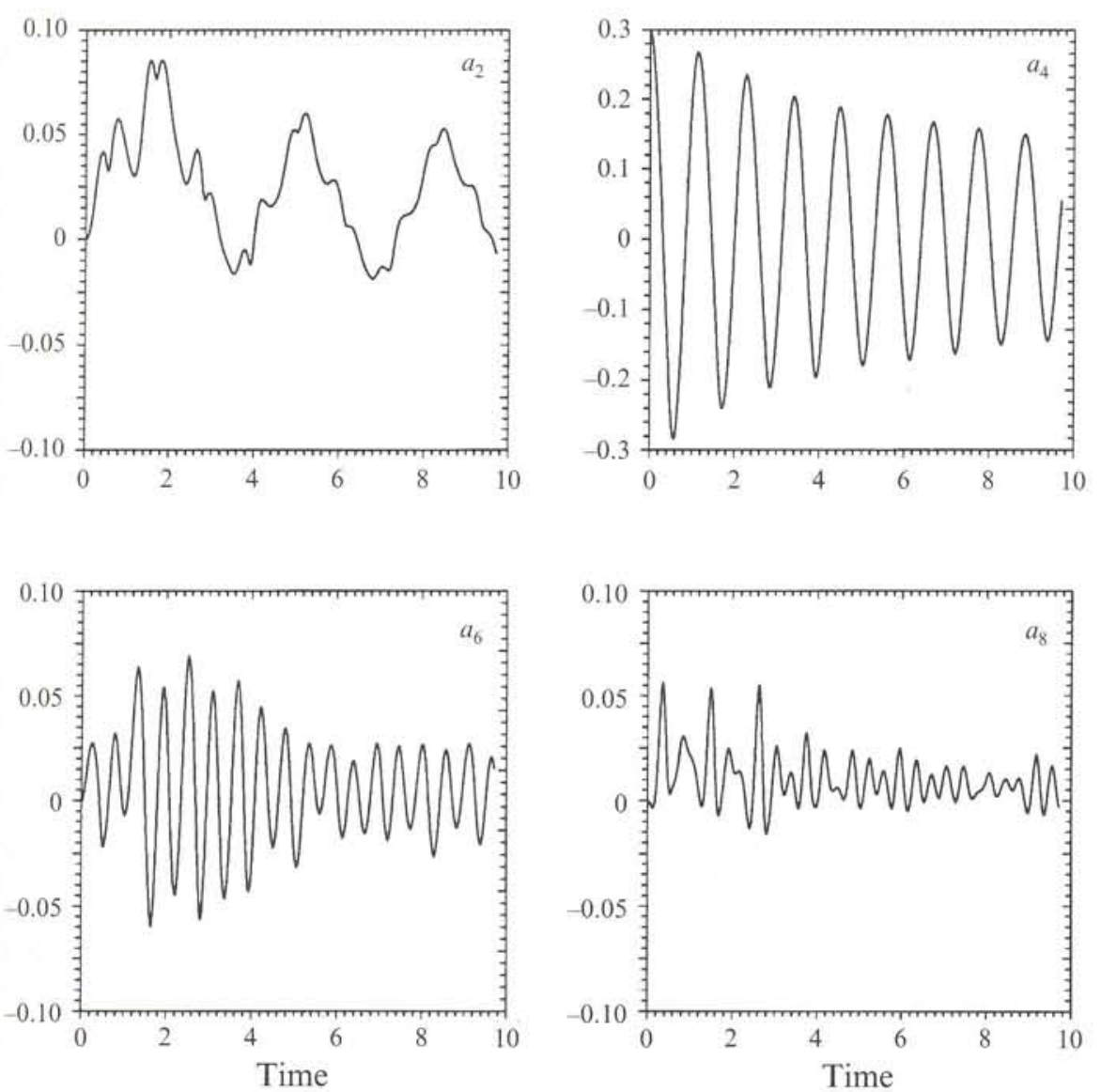

FIGURE 15. Calculated oscillations with weak viscous effects-compare with figures 7 and 8 of Lundgren \& Mansour (1989). The dimensionless viscosity of $v=4.0825 \times 10^{-4} r_{0}^{2} / T_{0}$ corresponds to their Reynolds number of 2000 . The time is scaled with $(3 / 2)^{\frac{1}{2}} T_{0}$. The other model parameters are given in the text.

Computations performed for several initial conditions (taken from experiments with ethanol and water droplets) indicate that these nonlinear couplings are in most cases the same and are approximately given by $C_{1} \approx 0.45$ and $C_{2} \approx 0.9$. The phenomena can be understood in terms of driving forces proportional to $a_{2}^{2}$ and $a_{2} a_{3}$ which are present in the differential equations for $a_{4}$ and $a_{5}$. The structures of these nonlinearity terms also follow from parity.

Asymptotically the higher modes are always forced oscillators, i.e. despite diminishing oscillation amplitudes the higher modes do not reach their linear solution (cf. figure 11). This is because the damping increases with the wavenumber $l$ (cf. (3.8)), and within a short time the higher modes become dependent solely on the energy transferred from the fundamental mode. Such a mode locking mechanism, selected by the linear damping constants, has already been described in Haken (1990, pp. 211-217).

\subsection{Additional computations and accuracy}

As was shown in the previous sections, it is typical in experimental observations that the lower modes $(I=2,3)$ contain most of the energy and that they are more or less the only degrees of freedom of surface motion. The higher modes, of lower energy, are in 


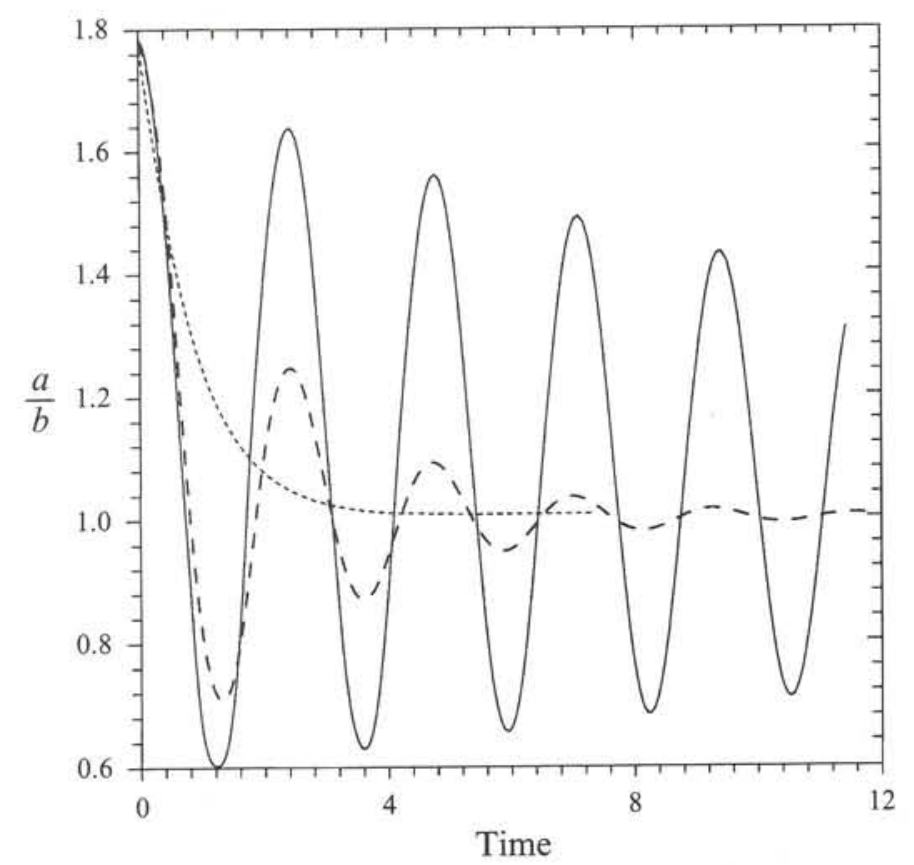

FIGURE 16. Transition from underdamped to critically damped conditions - compare with figure 12 of Basaran (1992). Variation of the droplet's prolate aspect ratio $a / b=R(0, t) / R\left(\frac{1}{2} \pi, t\right)$. The time is scaled with $\sqrt{ } 3 T_{0}$. Calculations for three dimensionless viscosities (Reynolds numbers): $v=5.7735 \times 10^{-3} r_{0}^{2} / T_{0} \quad(\operatorname{Re}=100) \quad\left(\right.$ solid line), $v=5.7735 \times 10^{-2} r_{0}^{2} / T_{0} \quad(R e=10) \quad($ dashed line), $\nu=5.7735 \times 10^{-1} r_{0}^{2} / T_{0}(R e=1)$ (dotted line). The initial conditions are given in the text. The other model parameters are $i_{0}=4, l_{0}=6$ and $\epsilon=0.1 r_{0}$.

fact generated by strong nonlinear coupling with $a_{2}$ and $a_{3}$. Such energy distributions, clearly noticeable in the damping constants of Lamb's approximation (3.8), seem to be typical of natural droplet oscillations. Of course, one might propose any artificial initial condition.

One interesting example with very low viscosity has been given by Lundgren \& Mansour (1989, figures 7 and 8). Their Reynolds number of 2000 corresponds to a dimensionless viscosity of $\nu=4.0825 \times 10^{-4} r_{0}^{2} / T_{0}$. We have repeated their calculation with $i_{0}=3, l_{0}=8$ and $\epsilon=0.1 r_{0}$ using their initial conditions, i.e.

$$
a_{4}(0)=0.3, \dot{a}_{4}(0)=0
$$

and zero for the other surface modes. The computed trajectories (figure 15) coincide quite well with those of Lundgren \& Mansour, except for a slight discrepancy in the extreme values of the amplitudes. The nonlinear characteristics are the same in both calculations: second-order coupling of $a_{2}$ and $a_{6}$ and third-order coupling of $a_{8}$ with the energy carrier $a_{4}$.

Another comparison is shown in figure 16. We have repeated calculations of Basaran (1992, figure 12) for a droplet starting to oscillate with only the second mode excited, i.e. $a_{2}(0)=0.4, \dot{a}_{2}(0)=0$ and zero for the other surface modes. The computations have been performed for three difference viscosities (Basaran's Reynolds numbers 100, 10 and 1), demonstrating transition from damped oscillations to an aperiodic decay.

It is worth mentioning that all of Basaran's nonlinear calculations showed aperiodic decay beyond a critical Reynolds number which closely corresponds to the critical 
value given by the linear theory. This justifies our approach of describing nonlinear droplet oscillations in terms of the linear modes.

The accuracy of our numerical solutions depends on the truncation numbers $i_{0}, l_{0}$ and the assumed thickness of the vortex layer $\left(r_{0}-\epsilon\right)$. It is rather difficult to find parameters justifying these values a priori. To find the optimal truncation numbers and the optimal radius $\epsilon$ several control calculations have been performed to analyse the influence of these parameters on the solution and its minimization errors (Becker 1991). It was found that for the cases analysed optimal values of $i_{0}$ and $l_{0}$ are 3 and 6 , respectively. Larger truncation numbers involve long computation times without significant improvement of the resulting accuracy. The radius $\epsilon$ of the zero-vorticity domain could be varied from $0.05 r_{0}$ to $0.3 r_{0}$ without noticeable deviations of the generated solutions.

\section{Concluding remarks}

A new droplet model for nonlinear viscous oscillations has been developed. The method is based on mode expansions with modified solutions of the linear problem and the application of the variational principle of Gauss. Computational results are in accordance with experimental data and numerical calculations of other authors up to relative droplet deformations of $80 \%$ of the equilibrium radius. Typical nonlinear characteristics like frequency modulation and mode coupling are found to be dominant even in the case of small deformations. Consequently, considerable discrepancies between the predictions of linear theory and the nonlinear dynamics are observed.

The present droplet model cannot describe such strong effects as droplet rupture. Also some fine details of the internal flow may become difficult to accurately model. However, our main interest, namely experimentally observable droplet deformations, are properly described over a wide range of the excitation amplitudes. The method also allow a wide variation of the dimensionless viscosity (oscillation Reynolds number), describing both aperiodic decay of droplet deformations and oscillations with nearly vanishing damping. This flexibility cannot be obtained easily by the available pure numerical methods.

The evaluation of surface and volume integrals at every time step involves relatively long computation times, limiting the applicability in solving practical problems. However, it has been found that with the help of the present model it is possible to construct simple differential equations which approximate the nonlinear behaviour of the surface parameters $a_{l}(t)$ and reduce the computational time by two orders of magnitude. Manipulating the coefficients of these equations, i.e. surface tension and viscosity, it is feasible to obtain within a short time an adequate description of the selected experimental data. Such a model can be easily applied to measure dynamic surface tension and viscosity by the oscillating droplet method.

This research was partially supported by the Deutsche Forschungsgemeinschaft (DFG). The authors wish to thank Priv.-Doz. Dr U. Brosa, Professor Dr F. Obermeier and Dr A. Dillmann for their suggestions and valuable discussions.

\section{Appendix A}

Looking at the linear solution given in $\S 3$, we can see the following problem. How can the amplitudes of the vorticity field $B_{i l}(3.15)$ be calculated if only the surface amplitude $a_{l}$ and the velocity field $v_{l}$ are given a priori? This question is not a trivial 
one. Consider a droplet at rest which starts to oscillate due to an initial surface deformation. To solve this special problem we must know a non-trivial set of amplitudes $B_{i l}$ that, combined with the velocities $\boldsymbol{b}_{i l}+c_{i l}^{0} \boldsymbol{c}_{l}$, assure a zero velocity field inside the droplet. Obviously these amplitudes remain unknown without further analysis. The reason is that any initial value problem is solved by mode analysis only if the system of equations is self-adjoint, i.e. if all its eigenvalues are real.

Let us apply Brosa's $(1986,1988)$ separation ansatz (B 3) in the following form:

$$
v_{l}(r, \theta, t)=\boldsymbol{\nabla} \times \boldsymbol{\nabla} \times\left\{\boldsymbol{r} B(r, t) P_{l}(\cos \theta)\right\}+c_{l}(t) \boldsymbol{\nabla}\left\{\left(r / r_{0}\right)^{l} P_{l}(\cos \theta)\right\} .
$$

The corresponding representation of the vorticity yields

$$
\boldsymbol{\nabla} \times \boldsymbol{v}_{l}(r, \theta, t)=-\frac{1}{\nu} \partial_{t} B(r, t) P_{l}^{\prime}(\cos \theta) \sin \theta \boldsymbol{e}_{\phi} .
$$

Thus an irrotational initial condition is given by

$$
a_{l}(t=0), \quad \dot{a}_{l}(t=0), \quad B(r, t=0)=0 .
$$

Applying (A 1) to the linearized boundary conditions we obtain

$$
\begin{gathered}
\frac{2 l\left(l^{2}-1\right) v}{r_{0}^{3}}\left(\partial_{r} B\left(r_{0}, t\right)-\frac{l}{r_{0}} B\left(r_{0}, t\right)\right)+\ddot{a}_{l}(t)+2 \delta \dot{a}_{l}(t)+\Omega^{2} a_{l}(t)=0, \\
\frac{1}{v} \partial_{t} B\left(r_{0}, t\right)=\frac{2}{r_{0}^{2}}\left(r_{0} \partial_{r} B\left(r_{0}, t\right)-l B\left(r_{0}, t\right)\right)-\frac{2(l-1)}{l} \dot{a}_{l}(t),
\end{gathered}
$$

where the kinematic boundary condition has been used to eliminate $c_{l}$ and $\dot{c}_{l}$. Because time and radius dependence have not been separated, the function $B(r, t)$ has to satisfy the diffusion equation

$$
\nu\left\{\partial_{r}^{2} B(r, t)+\frac{2}{r} \partial_{r} B(r, t)-\frac{l(l+1)}{r^{2}} B(r, t)\right\}=\partial_{t} B(r, t)
$$

which follow from the vorticity equation.

The dynamic boundary condition (A 4) ensures that for short times and irrotational initial conditions every droplet oscillation obeys Lamb's approximation (3.8). This holds independently of the value of $v$. As the droplet starts to oscillate, vorticity is generated at the surface owing to the tangential stress condition (A 5). For low fluid viscosity the vorticity cannot diffuse inside the droplet (the left side of (A 6) diminishes), and Lamb's approximation holds during the full oscillation time. This means that an irrotational initial condition is approximated by the weakly damped modes in the asymptotic limit of large Reynolds number $\left|\alpha^{2}\right|$. For larger viscosities the initial condition consists of both weakly damped and strongly dissipative modes. The latter disappear quickly in time. Hence, as already shown by Prosperetti (1977), the long-time behaviour of the droplet will differ from the initial one.

The analytical solution of the initial value problem (A 3)-(A 6) can be found using the standard Laplace transform method with the following conventions for a time function $f$ and its Laplace transform $\tilde{f}$ :

$$
\tilde{f}(\lambda)=\int_{0}^{\infty} f(t) \mathrm{e}^{\lambda t} \mathrm{~d} t, \quad \operatorname{Re}(\lambda)<0,
$$




$$
\begin{aligned}
f(t) & =\frac{-1}{2 \pi \mathrm{i}} \int_{\operatorname{Re}(\lambda)+\mathrm{i} \infty}^{\operatorname{Re}(\lambda)-\mathrm{i} \infty} \tilde{f}(\lambda) \mathrm{e}^{-\lambda t} \mathrm{~d} \lambda, \quad \operatorname{Re}(\lambda)<0, \\
& =-\sum \operatorname{Res}\left\{\tilde{f}(\lambda) \mathrm{e}^{-\lambda t}\right\}+\frac{1}{2 \pi \mathrm{i}} \int_{\nu} \tilde{f}(\lambda) \mathrm{e}^{-\lambda t} \mathrm{~d} \lambda .
\end{aligned}
$$

The sum in the inversion formula (A $8 b$ ) includes all residues of $\tilde{f}(\lambda) \mathrm{e}^{-\lambda t}$ with nonnegative real parts of $\lambda$. The integral of $(\mathrm{A} 8 b)$ represents the counterclockwise integration along the infinite semicircle with positive real parts.

Laplace transform of the system (A 4)-(A 6) with respect to the initial conditions (A 3) yields after several algebraic transformations:

$$
\begin{gathered}
\tilde{a}_{l}(\lambda)=-\frac{a_{l}(0)}{\lambda}-2 \frac{r_{0}^{2} / v}{\operatorname{det}(x)}\left\{\frac{\dot{a}_{l}(0)}{\lambda}+\frac{a_{l}(0) \Omega^{2}}{\lambda^{2}}\right\}\left\{\frac{\mathrm{d}\left(x_{j l}(x)\right)}{\mathrm{d} x}+\left(\frac{1}{2} x^{2}-(l+1)\right) j_{l}(x)\right\}, \\
\tilde{B}(r, \lambda)=\frac{2(l-1) r_{0}^{4}}{l v} \frac{j_{l}\left(x r / r_{0}\right)\left\{\dot{a}_{l}(0)+a_{l}(0) \Omega^{2} / \lambda\right\}}{\operatorname{det}(x)} .
\end{gathered}
$$

As expected the singularities of $\tilde{a}_{l}$ and $\tilde{B}$ coincide with the solutions of the characteristic equation (3.4). A formula equivalent to (A 9) has already been given by Prosperetti (1977) who used a numerical method to obtain the inverse transform. To invert (A 9) and (A 10) analytically we make use of (A $8 b)$ and obtain

$$
\begin{aligned}
& a_{l}(t)=\sum_{i=1}^{\infty} \frac{4 r_{0}^{2}}{v} \frac{\left(\dot{a}_{l}(0)+a_{l}(0) \Omega^{2} / \lambda_{i l}\right)\left(x_{i l} j_{l}^{\prime}\left(x_{i l}\right)+\left(x_{i l}^{2} / 2-l\right) j_{l}\left(x_{i l}\right)\right)}{x_{i l} \operatorname{det}^{\prime}\left(x_{i l}\right)} \mathrm{e}^{-\lambda_{i l} t} \\
& + \begin{cases}-\frac{1}{2} a_{t}(0), & t=0 \\
0, & t>0\end{cases} \\
& B(r, t)=a_{l}(0) \frac{2(l-1)(2 l+3) v}{l(2 l+1)}\left(\frac{r}{r_{0}}\right)^{l} \\
& -\sum_{i=1}^{\infty} \frac{4 r_{0}^{4}}{\nu} \frac{l-1}{l} \frac{\dot{a}_{l}(0) \lambda_{i}+a_{l}(0) \Omega^{2}}{x_{i l} \operatorname{det}^{\prime}\left(x_{i l}\right)} \mathrm{e}^{-\lambda_{i t} t} j_{l}\left(x_{i l} \frac{r}{r_{0}}\right),
\end{aligned}
$$

where $j_{l}^{\prime}$ and $\operatorname{det}^{\prime}$ denote the derivatives of $j_{l}(x)$ and $\operatorname{det}(x)$.

The result (A 12) gives rise to the following interpretation: the velocity modes $\left[b_{i l}+c_{i l}^{0} c_{l} \mid l=i \ldots \infty\right\}$ are linearly dependent for $l \geqslant 2$, i.e. there is one non-trivial combination of the amplitudes, namely

$$
B_{i l} \propto \frac{1}{b_{i l}^{0}} \frac{1}{x_{i l} \operatorname{det}^{\prime}\left(x_{i l)}\right)},
$$

resulting in zero velocity. Moreover (A 12) yields two non-trivial amplitude combinations for the vorticity modes $\left\{w_{i l} \mid i=1 \ldots \infty\right\}$ which generate zero vorticity for $r<r_{0}$, namely (A 13) and

$$
B_{i l} \propto \frac{1}{b_{i l}^{0}} \frac{x_{i l}}{\operatorname{det}^{\prime}\left(x_{i l}\right)} .
$$

However, if we consider only a finite number of velocity or vorticity modes, it turns out 
that they are linearly independent. For polar wavenumber $l=1$ the independence holds in general. This flexibility of the mode system confirms the completeness of Brosa's separation formulae.

\section{Appendix B}

In the case of constant fluid density and in the absence of bulk forces, partial solutions of the Stokes equation can be obtained as follows (Brosa 1986). The linearized Navier-Stokes equation

$$
\partial_{t} v(r, t)=-\frac{1}{\rho} \nabla p(r, t)-\nu \nabla \times \nabla \times v(r, t)
$$

and the equation of continuity

$$
\nabla \cdot v(r, t)=0
$$

are solved using the following representations:

$$
\begin{gathered}
v(\boldsymbol{r}, t)=\boldsymbol{\nabla} \times\{\boldsymbol{V} \beta(\boldsymbol{r}, t)\}+\boldsymbol{\nabla} \times \boldsymbol{\nabla} \times\{\boldsymbol{V} b(\boldsymbol{r}, t)\}+\boldsymbol{\nabla}\{c(\boldsymbol{r}, t)\}, \\
p(\boldsymbol{r}, t)=-\rho \partial_{t} c(\boldsymbol{r}, t),
\end{gathered}
$$

where the scalars $\beta, b$ and $c$ must satisfy the diffusion equations

$$
\nu \nabla^{2} \beta=\partial_{t} \beta, \quad \nu \nabla^{2} b=\partial_{t} b
$$

and the Laplace equation

$$
\nabla^{2} c=0,
$$

respectively. The supporting vector field $V$ is given by

$$
V=V_{0}+V_{1} r
$$

where $V_{0}$ and $V_{1}$ are arbitrary constants.

For the problem of droplet oscillations it is convenient to choose

$$
V=r \text {. }
$$

The partial solutions of (B 5) and (B 6) in spherical coordinates $(r, \theta, \phi)$ are given by (Moon \& Spencer 1961):

$$
\begin{gathered}
\beta \text { or } b \propto \mathrm{e}^{-\lambda t} j_{l}\left[(\lambda / v)^{\frac{1}{2}} r\right] Y_{l m}(\theta, \phi), \\
c \propto \mathrm{e}^{-\lambda t}\left(r / r_{0}\right)^{l} Y_{l m}(\theta, \phi) .
\end{gathered}
$$

$j_{l}$ denotes the spherical Bessel functions (Abramowitz \& Stegun, chap. 9) and $Y_{l m}$ the spherical harmonics. In the case of rotational symmetry $(m=0)$ the spherical harmonics can be substituted by the Legendre polynomials $P_{l}(\cos \theta)$.

The velocity fields corresponding to (B 9) and (B 10) have the following forms:

$$
\begin{aligned}
\nabla \times(r \beta) & =e_{\theta} \frac{\partial_{\phi} \beta}{\sin \theta}-e_{\phi} \partial_{\theta} \beta, \\
\nabla \times \nabla \times(r b) & =e_{r} \frac{l(l+1)}{r} b+e_{\theta} \partial_{\theta}\left(\partial_{r} b+\frac{b}{r}\right)+e_{\phi} \frac{\partial_{\phi}}{\sin \theta}\left(\partial_{r} b+\frac{b}{r}\right), \\
\nabla(c) & =e_{r} \frac{l}{r} c+e_{\theta} \frac{\partial_{\theta}}{r} c+e_{\phi} \frac{\partial_{\phi}}{r \sin \theta} c,
\end{aligned}
$$

where $e_{r}, \boldsymbol{e}_{\theta}$ and $\boldsymbol{e}_{\phi}$ are the unit vectors in the radial, polar and azimuthal directions. 
The tangential stresses of the velocity modes (B 11)-(B 13), evaluated on a sphere with radius $r_{0}$, are given by

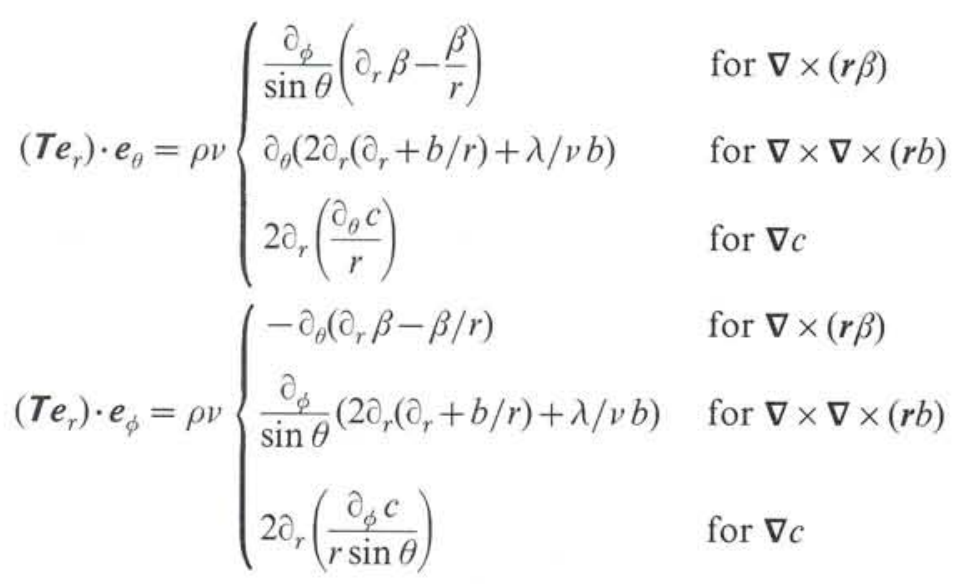

$\boldsymbol{T}$ denotes the Newtonian stress tensor of the corresponding velocity field. According to (B 14) and (B 15) the tangential stresses of $\boldsymbol{\nabla} \times \boldsymbol{\nabla} \times(\boldsymbol{r b})$ and $\boldsymbol{\nabla} c$ depend on the angles of $\theta$ and $\phi$ in the same way, namely proportional to $\partial_{\theta} Y_{l m}$ in the $\theta$-direction and proportional to $\left(\partial_{\phi} / \sin \theta\right) Y_{l m}$ in the $\phi$-direction. In the case of $\boldsymbol{\nabla} \times(\boldsymbol{r} \beta)$ these dependencies are exchanged with respect to the $(\theta, \phi)$-components. Hence, this velocity mode produces tangential forces on the surface that cannot be balanced by the other modes. The first term $\boldsymbol{\nabla} \times(\boldsymbol{r} \beta)$ of the representation formula (B 3) therefore vanishes if free boundary conditions of a liquid sphere are considered.

\section{REFERENCES}

Abramowitz, M. \& Stegun, I. A. 1968 Handbook of Mathematical Functions. Dover.

Amos, D. E. 1986 A portable package for Bessel functions of a complex argument and nonnegative order. ACM Trans. Math. Software 12, 265-273.

Basaran, O. A. 1992 Nonlinear oscillations of viscous liquid drops. J. Fluid Mech. 241, 169-198.

BECKER, E. 1991 Nichtlineare Tropfenschwingungen unter Berücksichtigung von Oberflächenspannung und Viskosität (Nonlinear oscillations of viscous droplets driven by surface tension). Mitteilungen aus dem Max-Planck-Institut für Strömungsforschung, vol. 104. E.-A. Müller MPI Göttingen.

Becker, E., Hiller, W. J. \& Kowalewski, T. A. 1991 Experimental and theoretical investigation of large-amplitude oscillations of liquid droplets. J. Fluid Mech. 231, 189-210.

Boberg, L. \& Brosa, U. 1988 Onset of turbulence in a pipe. Z. Naturforsch. 43a, 697-727.

Brosa, U. 1986 Linear analysis of the currents in a pipe. Z. Naturforsch. 41a, 1141-1153.

BrosA, U. 1988 Strongly dissipative modes. Unpublished paper, Universität Marburg.

BrosA, U. \& BECKer, E. 1988 Das zufällige Halsreißen (Random Neck Rupture). Movie C 1694, available (in German or English) on loan from the IWF, Nonnenstieg 72, D-37075 Göttingen, Germany.

Brosa, U., Grossmann, S., Müller, A. \& Becker, E. 1989 Nuclear scission. Nuclear Phys. A 502, $423 \mathrm{c}-442 \mathrm{c}$.

Chandrasekhar, S. 1961 Hydrodynamic and Hydromagnetic Stability. Clarendon.

Defay, R. \& PÉtré, G. 1971 Dynamic surface tension. In Surface and Colloid Science (ed. E. Matijević), pp. 27-81. Wiley-Interscience.

FeHLbERG, E. 1970 Klassiche Runge-Kutta-Formeln vierter und niedriger Ordnung mit Schrittweitenkontrolle und ihre Anwendung auf Wärmeleitungsprobleme. Computing 6, 61-71.

HAKEN, H. 1990 Synergetik, 3rd edn. Springer. 
Hiller, W. J. \& Kowalewski, T. A. 1989 Surface tension measurements by the oscillating droplet method. Phys. Chem. Hydrodyn. 11, 103-112.

LAMB, H. 1932 Hydrodynamics, 6th edn. Cambridge University Press.

LUNDGREN, T. S. \& MANSOUR, N. N. 1988 Oscillation of drops in zero gravity with weak viscous effects. J. Fluid Mech. 194, 479-510.

Moon, P. \& SPencer, D. E. 1961 Field Theory Handbook. Springer.

NATARAJAN, R. \& BRown, R. A. 1987 Third-order resonance effect and the nonlinear stability of drop oscillation. J. Fluid Mech. 183, 95-121.

Patzek, T. W., Brenner, R. E., Basaran, O. A. \& Scriven, L. E. 1991 Nonlinear oscillations of inviscid free drops. J. Comput. Phys. 97, 489-515.

Prosperetti, A. 1977 Viscous effects on perturbed spherical flows. Q. Appl. Maths 35, 339-352.

ProsperetTi, A. $1980 a$ Normal-mode analysis for the oscillations of a viscous liquid drop immersed in another liquid. J. Méc. 19, 149-182.

Prosperetti, A. $1980 \mathrm{~b}$ Free oscillations of drops and bubbles: the initial-value problem. $J$. Fluid Mech. 100, 333-347.

RAYleigh, LORD 1899 On the capillary phenomena of jets. Proc. R. Soc. Lond. A 29, 71-97.

STOER, J. 1972 Einführung in die Numerische Mathematik I. Springer.

StÜCKRAD, B., Hiller, W. J. \& KOWALEWSKI, T. A. 1993 Measurement of dynamic surface tension by the oscillating droplet method. Exp. Fluids (to appear).

Tsamopoulos, J. A. \& Brown, R. A. 1983 Nonlinear oscillations of inviscid drops and bubbles. J. Fluid Mech. 127, 519-537.

WILKENING, V. 1992 Betrachtungen im Hyperraum-'Relation' verarbeitet Multi-parameterDaten. c't 1, 70-74. 